\title{
Design and Multiplierless Realization of Digital Synthesis Filters for Hybrid-Filter-Bank A/D Converters
}

\author{
S. H. Zhao and S. C. Chan, Member, IEEE
}

\begin{abstract}
This paper studies the optimal least squares and minimax design and realization of digital synthesis filters for hybrid-filter-bank analog-to-digital converters (HFB ADCs) to meet a given spurious-free dynamic range (SFDR). The problem for designing finite-impulse-response synthesis filters is formulated as a second-order cone-programming problem, which is convex and allows linear and quadratic constraints such as peak aliasing error to be incorporated. The fixed coefficients of the designed synthesis filters are efficiently implemented using sum-of-power-of-two (SOPOT) coefficients, while the internal word length used for each intermediate data is minimized using geometric programming. The main sources of error are analyzed, and a new formula of SFDR in terms of these errors is derived. The effects of component variations of analog analysis filters on the HFB ADC are also addressed by means of two new robust HFB ADC design algorithms based on stochastic uncertainty and worst case uncertainty models. Design results show that the proposed approach offers more flexibility and better performance than conventional methods in achieving a given SFDR and that the robust design algorithms are more robust to parameter uncertainties than the nominal design in which the uncertainties are not taken into account.
\end{abstract}

Index Terms-Analog-to-digital converters (ADCs), hybrid filter bank (HFB), multiplierless realization, parameter uncertainty, second-order cone programming (SOCP), synthesis filter design.

\section{INTRODUCTION}

A HYBRID filter bank (HFB) is an efficient technique to increase the conversion speed of analog-to-digital converters (ADCs) and digital-to-analog converters (DACs) [1], [2]. Fig. 1 shows an $M$-channel HFB ADC, where a set of analysis filters $\left\{H_{m}(s)\right\}$ are used to channelize the wideband input signal into $M$ subband signals. Each subband signal is sampled and quantized at $1 / M$ times the effective sample rate of the system by an $n$-bit ADC in the ADC array. The digitized subband signals are upsampled by a factor of $M$ and then synthesized by means of a set of digital synthesis filters $\left\{F_{m}(z)\right\}$ to yield the reconstructed signal. Compared to ADCs using a standard time-interleaved array conversion technique

Manuscript received June 03, 2008; revised October 07, 2008. First published January 06, 2009; current version published September 30, 2009. This paper was presented in part at the 2006 IEEE International Symposium on Circuits and Systems [24]. This paper was recommended by Associate Editor Y. Lian.

The authors are with the Department of Electrical and Electronic Engineering, The University of Hong Kong, Kowloon, Hong Kong (e-mail: shzhao@eee. hku.hk; scchan@eee.hku.hk).

Digital Object Identifier 10.1109/TCSI.2008.2012213

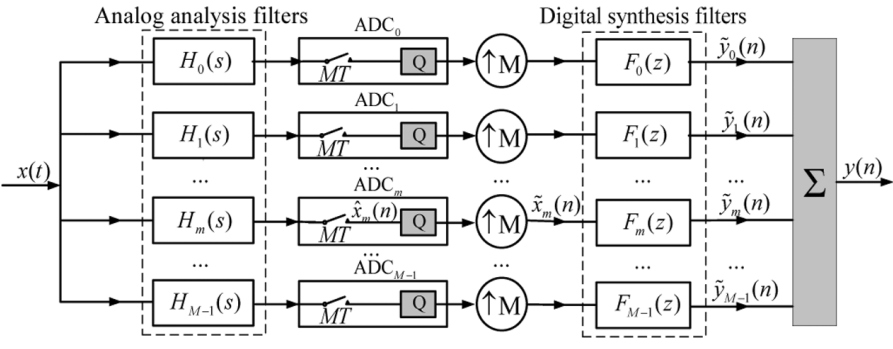

Fig. 1. Structure of an HFB ADC.

[3], HFB ADCs have the advantage of lower sensitivity to the effect of gain and phase mismatch between the converters, so the speed and resolution of conversion can be improved [4]. On the other hand, it requires a slightly more analog circuitry. Because of these reasons, HFB ADCs have been the subject of active research recently [2], [5]-[7]. In [2], an HFB ADC design method based on frequency-domain analysis was proposed. Analog analysis filters were designed by discrete- to continuous-time transformation. In particular, the designed discrete-time filter bank was converted into a continuous-time filter bank, and synthesis filters were designed using a fast Fourier transform. The major drawback of this approach is that it may lead to high-order continuous-time filters. In [5], a least squares (LS) error minimization method was introduced. In [6], a two-channel HFB ADC design algorithm was proposed. The coefficients of analog and digital filters were optimized to minimize the magnitude of the reconstruction errors using the minimax criterion.

In this paper, a new synthesis filter design algorithm for HFB ADCs using second-order cone programming (SOCP) [8] is proposed. In the proposed method, simple second-order analog Butterworth filters are employed as analysis filters so as to reduce the implementation complexity and to simplify the analog specifications. Other appropriate analog filters can also be used. Given the analysis filters, finite-impulse-response (FIR) filters are used to minimize the reconstruction errors. The problem of designing digital FIR synthesis filters is formulated as a convex optimization problem and solved using SOCP. SOCP is a kind of convex optimization problem, and the global optimal solution, if it exists, can be found. The ability of SOCP to impose linear and convex quadratic constraints allows the proposed design method to offer more flexibility in terms of design objectives such as the minimax and LS criteria and other design constraints such as peak limitations on aliasing errors and prescribed magnitude flatness on the reconstructed signal. This is of 
great importance in ADC design because the reconstruction errors can be masked below a certain level so that the accuracy of the entire ADC in terms of spurious-free dynamic range (SFDR) can conveniently be controlled. SFDR is an important measure of the accuracy of an ADC. It is defined as the ratio of the rootmean-square (rms) amplitude of the carrier frequency (maximum signal component) to the rms value of the next largest noise or harmonic-distortion component. The latter is governed by four main sources of error in implementing the HFB ADC, including the quantization noises from ADCs in the ADC array; the finite word-length effect, including the coefficient round-off noises and signal round-off noises; and the aliasing errors due to the HFB structure. These errors have to be masked below certain levels so that the desired SFDR can be achieved. In Section IV, a new relationship between these errors and the SFDR is derived to facilitate the design and realization of the HFB ADC.

As mentioned earlier, to satisfy a given SFDR, the peak reconstruction error at every frequency in the signal band of interest has to be masked below a certain level. This is somewhat difficult to achieve by a conventional digital synthesis filter design technique using the LS criterion [5]. Using the proposed SOCP design method, such objectives can readily be incorporated. This will be further illustrated by design examples later in Section V. Once the coefficients of digital synthesis filters are determined, the next important problems are to control the finite word length and signal round-off errors so that the given SFDR will still be satisfied. Since the designed synthesis filters have fixed filter coefficients, they can be implemented efficiently using sum-of-power-of-two (SOPOT) coefficients or canonical signed digit [11]-[14]. By using sufficient number of SOPOT terms, the reconstruction errors can be controlled to the desired level, even after coefficient quantization using these coefficients. As a result, the entire synthesis filters can be implemented without any multiplication. In addition, the redundancy in realizing the multiplications of these SOPOT coefficients can significantly be reduced by using the multiplier-block (MB) technique [15], which gives rise to minimum adder realization. As the signal round-off noise is dependent on the word length of the intermediate data, they should appropriately be chosen to meet the given SFDR specification while reducing the hardware resources. In this paper, the geometric-programming (GP) approach proposed in [18] is used to minimize all the internal word lengths in order to satisfy the given SFDR budget. As the hardware complexity such as adder cells and registers is closely related to the internal word lengths, such a hardware resource will also be minimized. To our best knowledge, such a systematic design approach in meeting a given SFDR specification is unavailable in the literature.

Another objective of this paper is to study and address the parameter variations of analog analysis filters and their effects on the reconstruction quality of the HFB ADC. Such variations may originate from different operation environments, component-value variations, interference, and aging. These variations may degrade the overall performance of the HFB ADC significantly. Therefore, when designing digital synthesis filters, these uncertainties or possible variations in analog analysis filters should be taken into account. Two robust methods, namely, the stochastic robust design (SRD) and the worst case robust de- sign (WCRD), are proposed to mitigate the effect of these uncertainties. The former minimizes the stochastic expectation of the design objective, and the latter regards the worst case error as the design objective. Design results show that these approaches are more robust to parameter variations introduced in analog analysis filters than conventional approaches that do not take the uncertainties into account.

The rest of this paper is organized as follows. Section II is devoted to the design of digital synthesis filters for the HFB ADC using the minimax and LS criteria. The problem of multiplierless realization and word-length determination of digital synthesis filters is discussed in Section III. Section IV analyzes the four main sources of errors in implementing HFB ADCs and presents a new relationship between the SFDR and these errors. Section V studies the robust HFB ADC design with component uncertainties. Design examples and performance evaluation of the proposed approaches are given in Section VI. Finally, conclusions are drawn in Section VII.

\section{Proposed Design Using SOCP}

\section{A. Analog Analysis Filters}

For HFB ADCs, passband ripples and nonconstant group delay of analog analysis filters usually require high-order digital synthesis filters in order to achieve an accurate signal reconstruction. Butterworth filters, which have a monotonic passband magnitude response without passband ripple and nearly constant group delay, are thus good candidates for constructing analysis filters. Therefore, Butterworth filters are chosen as analog analysis filters in this paper. Other analog filters can also be used. To reduce analog components, we shall consider second-order Butterworth filters, and they are designed so as to divide the input signal approximately into $M$ equally spaced subchannels.

\section{B. FIR Synthesis Filter Design Using SOCP}

For an $M$-channel HFB ADC, the result of filtering the input signal $X(j \Omega)$ through the $m$ th analog analysis filter $H_{m}(j \Omega)$ can be written as $X_{m}(j \Omega)=X(j \Omega) H_{m}(j \Omega)$. The filtered signal is then sampled at a rate of $1 /(M T)$, where $T$ is the sample period of the system. The sampled signal at the input of the $m$ th quantizer is then given by

$$
\begin{aligned}
& \hat{X}_{m}\left(e^{j \omega}\right) \\
& =\frac{1}{M T} \sum_{p=-\infty}^{+\infty} X\left(j \frac{\omega}{M T}-j \frac{2 \pi p}{M T}\right) H_{m}\left(j \frac{\omega}{M T}-j \frac{2 \pi p}{M T}\right)
\end{aligned}
$$

where $\omega=\Omega T$. After quantization, the digitized signals are upsampled and filtered by digital synthesis filters. The output of the $m$ th subchannel is given by

$$
\begin{aligned}
& \tilde{Y}_{m}\left(e^{j \omega}\right) \\
& =\frac{1}{M T} \sum_{p=-\infty}^{+\infty} X\left(j \frac{\omega}{T}-j \frac{2 \pi p}{M T}\right) H_{m}\left(j \frac{\omega}{T}-j \frac{2 \pi p}{M T}\right) F_{m}\left(e^{j \omega}\right)
\end{aligned}
$$


where $F_{m}\left(e^{j \omega}\right)$ is the digital synthesis filter of the $m$ th subchannel. The digitized signals from all subchannels are finally summed together to yield the reconstructed signal

$$
\begin{aligned}
Y\left(e^{j \omega}\right)=\frac{1}{M T} \sum_{m=0}^{M-1} \sum_{p=-\infty}^{+\infty} X\left(j \frac{\omega}{T}-j \frac{2 \pi p}{M T}\right) \\
\times H_{m}\left(j \frac{\omega}{T}-j \frac{2 \pi p}{M T}\right) F_{m}\left(e^{j \omega}\right) .
\end{aligned}
$$

Assuming that the input signal is band limited to $\pi / T$ by certain external filters, only the terms with $p=0, \ldots, M-1$ in the inner summation of (1) remain, and it gives

$$
Y\left(e^{j \omega}\right)=\sum_{p=0}^{M-1} X\left(j \frac{\omega}{T}-j \frac{2 \pi p}{M T}\right) T_{p}\left(e^{j \omega}\right)
$$

where

$$
T_{p}\left(e^{j \omega}\right)=\frac{1}{M T} \sum_{m=0}^{M-1} F_{m}\left(e^{j \omega}\right) H_{m}\left(j \frac{\omega}{T}-j \frac{2 \pi p}{M T}\right) .
$$

It can be seen from (2) that the output signal is a mixture of the frequency-shifted versions of the input signal. $T_{p}\left(e^{j \omega}\right)$ represents the transfer functions of the HFB ADC. $T_{0}\left(e^{j \omega}\right)$ is the distortion function representing the magnitude gain and groupdelay functions of the HFB, and $T_{p}\left(e^{j \omega}\right), p=1, \ldots, M-1$, denotes the aliasing function associated with the shifted and unwanted versions of the input signal that should ideally be eliminated. An ideal reconstruction result is obtained if the reconstructed signal is a scaled and delayed version of the input signal, which is referred to as the perfect-reconstruction (PR) condition [1]. The ideal transfer function of an HFB ADC is

$$
D_{p}\left(e^{j \omega}\right)= \begin{cases}c e^{-j \omega d}, & p=0 \\ 0, & p=1, \ldots, M-1\end{cases}
$$

where $c$ is a nonzero constant and $d$ is the system delay. The difference between the ideal transfer function and the real transfer function, namely, the approximation error, is given by

$$
e_{p}(\omega)=T_{p}\left(e^{j \omega}\right)-D_{p}\left(e^{j \omega}\right) .
$$

Synthesis filters should be designed so as to minimize the approximation error using a certain criterion. The design of FIR synthesis filters is considered first. Assume that the $m$ th synthesis filter is an $N$-tap FIR filter with $z$-transform $F_{m}(z)=\sum_{n=0}^{N-1} f_{m}(n) z^{-n}$, where $f_{m}(n)$ is the coefficient of the $m$ th synthesis filter to be determined. Its frequency response $F_{m}\left(e^{j \omega}\right)$ is given by

$$
F_{m}\left(e^{j \omega}\right)=\sum_{n=0}^{N-1} f_{m}(n) e^{-j n \omega}=\boldsymbol{f}_{m}^{T}\{\boldsymbol{c}(\omega)-j \boldsymbol{s}(\omega)\}
$$

where $f_{m}=\left[f_{m}(0), \ldots, f_{m}(N-1)\right]^{\mathrm{T}}, \boldsymbol{c}(\omega)=$ $[1, \cos (\omega), \ldots, \cos ((N-1) \omega)]^{\mathrm{T}}, \quad$ and $\boldsymbol{s}(\omega)=$ $[0, \sin (\omega), \ldots, \sin ((N-1) \omega)]^{\mathrm{T}}$. Using FIR synthesis filters, the transfer function of the HFB ADC can be written as

$T_{p}\left(e^{j \omega}\right)=\frac{1}{M T} \sum_{m=0}^{M-1} \boldsymbol{f}_{m}^{T}\{\boldsymbol{c}(\omega)-j \boldsymbol{s}(\omega)\} H_{m}\left(j \frac{\omega}{T}-j \frac{2 \pi p}{M T}\right)$.

To approximate the PR condition in the minimax sense, we solve the following min-max problem:

$\min _{\boldsymbol{f}}\left\{\max W_{p}(\omega)\left|e_{p}(\omega)\right|\right\}, \quad \omega \in \Omega_{I}, \quad p=0, \ldots, M-1$

where $f=\left[\boldsymbol{f}_{0}^{T}, \boldsymbol{f}_{1}^{T}, \ldots, \boldsymbol{f}_{M-1}^{T}\right]^{\mathrm{T}}, \Omega_{I}$ is the frequency band of interest, and $W_{p}(\omega)$ is a positive weighting parameter specifying the relative importance between the distortion and aliasing errors. The objective in (5) can be written as

$$
\begin{aligned}
& W_{p}(\omega)\left|e_{p}(\omega)\right| \\
& \quad=W_{p}(\omega)\left|T_{p}\left(e^{j \omega}\right)-D_{p}\left(e^{j \omega}\right)\right| \\
& \quad=W_{p}(\omega) \|\left[\begin{array}{l}
\left.\left.\left.\left[\boldsymbol{r}_{p, 0}^{T}(\omega), \ldots, \boldsymbol{r}_{p, M-1}^{T}(\omega)\right] \boldsymbol{f}-R_{p}^{\mathrm{d}}(\omega)\right] \boldsymbol{i}_{p, 0}^{T}(\omega), \ldots, \boldsymbol{i}_{p, M-1}^{T}(\omega)\right] \boldsymbol{f}-I_{p}^{\mathrm{d}}(\omega)\right]
\end{array} \|_{2}\right. \\
& \quad=W_{p}(\omega)\left[\left(\boldsymbol{r}_{p}^{T}(\omega) \boldsymbol{f}-R_{p}^{\mathrm{d}}(\omega)\right)^{2}+\left(\boldsymbol{i}_{p}^{T}(\omega) \boldsymbol{f}-I_{p}^{\mathrm{d}}(\omega)\right)^{2}\right]^{1 / 2} \\
& \quad=\left[\left(\alpha_{p}^{\mathrm{R}}(\omega)\right)^{2}+\left(\alpha_{P}^{I}(\omega)\right)^{2}\right]^{1 / 2}
\end{aligned}
$$

where

$$
\begin{aligned}
R_{p}^{\mathrm{d}}(\omega) & =\left[D_{p}\left(e^{j \omega}\right)\right]_{R} \quad I_{p}^{\mathrm{d}(}(\omega)=\left[D_{p}\left(e^{j \omega}\right)\right]_{\mathrm{I}} \\
\boldsymbol{r}_{p, m}(\omega) & =\left[\frac{1}{M T} H_{m}\left(j \frac{\omega}{T}-j \frac{2 \pi p}{M T}\right)(\boldsymbol{c}(\omega)-j \boldsymbol{s}(\omega))\right]_{R} \\
\boldsymbol{i}_{p, m}(\omega) & =\left[\frac{1}{M T} H_{m}\left(j \frac{\omega}{T}-j \frac{2 \pi p}{M T}\right)(\boldsymbol{c}(\omega)-j \boldsymbol{s}(\omega))\right]_{I} \\
\boldsymbol{r}_{p}(\omega) & =\left[\boldsymbol{r}_{p, 0}^{T}(\omega), \ldots, \boldsymbol{r}_{p, M-1}^{T}(\omega)\right]^{\mathrm{T}} \\
\boldsymbol{i}_{p}(\omega) & =\left[\boldsymbol{i}_{p, 0}^{T}(\omega), \ldots, \boldsymbol{i}_{p, M-1}^{T}(\omega)\right] \\
\alpha_{p}^{\mathrm{R}}(\omega) & =W_{p}(\omega)\left[\boldsymbol{r}_{p}^{T}(\omega) \boldsymbol{f}-R_{p}^{\mathrm{d}}(\omega)\right] \\
\alpha_{P}^{I}(\omega) & =W_{p}(\omega)\left[\boldsymbol{i}_{p}^{T}(\omega) \boldsymbol{f}-I_{p}^{\mathrm{d}}(\omega)\right] .
\end{aligned}
$$

Here, $[\cdot]_{R}$ and $[\cdot]_{I}$ return the real and imaginary parts of a complex number or vector, respectively, and $\|\boldsymbol{x}\|_{2}$ is the two- or Euclidean norm of a vector $\boldsymbol{x}$. Hence, (5) can be reformulated as

$$
\begin{array}{cl}
\min _{f} & \delta \\
\text { subject to } & \delta-\left[\left(\alpha_{p}^{\mathrm{R}}(\omega)\right)^{2}+\left(\alpha_{P}^{\mathrm{I}}(\omega)\right)^{2}\right]^{1 / 2} \geq 0 .
\end{array}
$$

Discretizing the frequency variable $\omega$ over a dense set of frequencies $\left\{\omega_{i}, 1 \leq i \leq K_{l}\right\}$ on the frequency band of interest, the constraints become $\delta-\left[\left(\alpha_{p}^{\mathrm{R}}\left(\omega_{i}\right)\right)^{2}+\left(\alpha_{P}^{\mathrm{I}}\left(\omega_{i}\right)\right)^{2}\right]^{1 / 2} \geq 0$. 
Then, (6) can be cast to the following SOCP problem, which can readily be solved using a standard SOCP solver

$$
\begin{array}{cl}
\min _{\boldsymbol{x}} & \boldsymbol{c}^{T} \boldsymbol{x} \\
\text { subject to } & \boldsymbol{c}^{T} \boldsymbol{x} \geq\left\|\boldsymbol{F}_{p, i} \boldsymbol{x}-\boldsymbol{g}_{p, i}\right\|_{2}
\end{array}
$$

where $\boldsymbol{c}=\left[1, \boldsymbol{O}_{N M}^{T}\right]^{\mathrm{T}}, \boldsymbol{x}=\left[\delta, \boldsymbol{f}^{T}\right]^{\mathrm{T}}, \boldsymbol{F}_{p, i}=$ $W_{p}\left(\omega_{i}\right)\left[\begin{array}{rr}0 & \boldsymbol{r}_{p}^{T}\left(\omega_{i}\right) \\ 0 & \boldsymbol{i}_{p}^{T}\left(\omega_{i}\right)\end{array}\right], \boldsymbol{g}_{p, i}=W_{p}\left(\omega_{i}\right)\left[R_{p}^{\mathrm{d}}\left(\omega_{i}\right), I_{p}^{\mathrm{d}}\left(\omega_{i}\right)\right]^{\mathrm{T}}$, $p=0, \ldots, M-1$, and $1 \leq i \leq K_{l} . O_{N}$ is an $N$-row zero vector.

Instead of the minimax criterion, the LS error criterion

$$
E_{\mathrm{LS}}(\boldsymbol{f})=\sum_{p=0}^{M-1} \int_{\Omega_{\mathrm{I}}} W_{p}(\omega)\left|T_{p}\left(e^{j \omega}\right)-D_{p}\left(e^{j \omega}\right)\right|^{2} \mathrm{~d} \omega
$$

can also be minimized. Equation (8) can be rewritten in matrix form as

$$
\begin{aligned}
E_{\mathrm{LS}}(\boldsymbol{f}) & =\boldsymbol{f}^{T} \boldsymbol{S} \boldsymbol{f}-2 \boldsymbol{f}^{T} \boldsymbol{v}+k \\
& =\left\|\boldsymbol{S}^{1 / 2} \boldsymbol{f}-\boldsymbol{S}^{-1 / 2} \boldsymbol{v}\right\|_{2}^{2}+\left(\boldsymbol{v}^{T} \boldsymbol{S}^{-1} \boldsymbol{v}-k\right)
\end{aligned}
$$

where

$$
\begin{aligned}
\boldsymbol{S} & =\sum_{p=0}^{M-1} \int_{\Omega_{\mathrm{I}}} W_{p}(\omega)\left[\boldsymbol{r}_{p}(\omega) \boldsymbol{r}_{p}^{T}(\omega)+\boldsymbol{i}_{p}(\omega) \boldsymbol{i}_{p}^{T}(\omega)\right] \mathrm{d} \omega \\
\boldsymbol{v} & =\sum_{p=0}^{M-1} \int_{\Omega_{\mathrm{I}}} W_{p}(\omega)\left[\boldsymbol{r}_{p}(\omega) R_{p}^{d}(\omega)+\boldsymbol{i}_{p}(\omega) I_{p}^{d}(\omega)\right] \mathrm{d} \omega \\
k & =\sum_{p=0}^{M-1} \int_{\Omega_{\mathrm{I}}} W_{p}(\omega)\left|D_{p}\left(e^{j \omega}\right)\right|^{2} \mathrm{~d} \omega .
\end{aligned}
$$

To design the FIR synthesis filters using the LS criterion, we solve the following SOCP problem:

$$
\begin{array}{cl}
\min _{\boldsymbol{x}} & \boldsymbol{c}^{T} \boldsymbol{x} \\
\text { subject to } & \boldsymbol{c}^{T} \boldsymbol{x} \geq\|\hat{\boldsymbol{S}} \boldsymbol{x}-\hat{\boldsymbol{v}}\|_{2}
\end{array}
$$

where $\hat{\boldsymbol{S}}=\left[\boldsymbol{O}_{N M}, \boldsymbol{S}^{-1 / 2}\right]$ and $\hat{\boldsymbol{v}}=S^{-1 / 2} \boldsymbol{v}$.

The filter length $N$ of digital synthesis filters is an important parameter that determines the system performance. A longer filter length gives smaller distortion error and larger aliasingerror attenuation at the expense of increased system complexity. As mentioned in the Introduction, SFDR is an important parameter to evaluate the performance of ADCs. The relationship between the system resolution and SFDR is given by $S F D R_{\text {bits }}=$ $S F D R(\mathrm{dBc}) / 6.02$, where $S F D R_{\text {bits }}$ is the effective number of bits [19]. For a given system resolution in $S F D R_{\text {bits }}$, the filter length in the proposed algorithm is chosen in such a way that the distortion error is reduced to less than a tenth of a decibel deviation from the ideal $0 \mathrm{~dB}$, and the aliasing errors are canceled to a sufficiently low level so that, after incorporating the quantization noises from ADCs and the finite word-length effect, it will still be lower than the desired SFDR (i.e., 6.02 .
$\left.S F D R_{\text {bits }}\right)$. This will be discussed later in Section IV. Usually, a peak aliasing error with a few decibels lower than the desired SFDR is chosen. Then, the minimum synthesis filter length that satisfies the specification can be determined by incorporating the desired SFDR as additional convex quadratic inequalities in the SOCP framework earlier. The detailed formulation will be illustrated later in Section II-C.

Note that, for systems requiring lower reconstruction delay, the length of FIR synthesis filters can be very long. In this case, IIR filters serve as a good alternative to offer more flexibility and lower arithmetic complexity. With the design framework described earlier, we proposed in [25] to design IIR synthesis filters using the model-reduction technique in [9] and [10]. The resulting IIR synthesis filters would have a much lower filter order than their FIR counterparts, particularly for low-delay systems. More details can be found in [25].

\section{Linear and Convex Quadratic Constraints}

1) Imposing linear equality constraints: Magnitude flatness at certain frequency points in the distortion function is sometimes desirable in HFB ADC design. It can be formulated as an additional linear equality constraint in the SOCP framework.

Consider the following relation between the derivatives of the design frequency response and its ideal counterparts:

$$
\left.\frac{\mathrm{d}^{i}}{\mathrm{~d} z^{i}} T_{0}\left(e^{j \omega}\right)\right|_{\omega=\widehat{\omega}}=\left.\frac{\mathrm{d}^{i}}{\mathrm{~d} z^{i}} D_{0}\left(e^{j \omega}\right)\right|_{\omega=\widehat{\omega}}, \quad i=0, \ldots, K_{\mathrm{L}}-1 .
$$

Equation (11) tells us that the system to be designed should approximate the desired response at $\omega=\widehat{\omega}$ up to the $\left(K_{\mathrm{L}}-1\right)$ th order. To impose a magnitude flatness on the order of $K_{\widehat{\omega}_{d}}-1$ on $T_{0}\left(e^{j \omega}\right)$ at frequency $\widehat{\omega}_{d}$, we have

$$
\begin{array}{r}
\frac{1}{M T} \sum_{m=0}^{M-1} \sum_{n=0}^{N-1} H_{m}\left(j \frac{\widehat{\omega}_{d}}{T}\right)(n-d)^{u} f_{m}(n) e^{-j \widehat{\omega}_{d}(n-d)} \\
= \begin{cases}c, & u=0 \\
0, & u=1, \ldots, K_{\widehat{\omega}_{d}}-1 .\end{cases}
\end{array}
$$

Equation (12) can be written in matrix form as

$$
\boldsymbol{L}_{\widehat{\omega}_{d}} \cdot \boldsymbol{f}=\boldsymbol{d}_{\widehat{\omega}_{d}}
$$

where

$$
\begin{aligned}
\boldsymbol{L}_{\widehat{\omega}_{d}}= & {\left[\boldsymbol{l}_{\widehat{\omega}_{d}, 0}^{T}, \ldots, \boldsymbol{l}_{\widehat{\omega}_{d}, K_{\hat{\omega}_{d}}-1}^{T}\right]^{\mathrm{T}} } \\
\boldsymbol{l}_{\widehat{\omega}_{d}, u}= & {\left[\boldsymbol{l}_{\widehat{\omega}_{d}, u}^{0}, \ldots, \boldsymbol{l}_{\widehat{\omega}_{d}, u}^{M-1}\right] } \\
\boldsymbol{l}_{\widehat{\omega}_{d}, u}^{m}= & {\left[\frac{1}{M T} H_{m}\left(j \frac{\widehat{\omega}_{d}}{T}\right)(0-d)^{u} e^{-j \widehat{\omega}_{d}(0-d)}, \ldots,\right.} \\
& \left.\quad \frac{1}{M T} H_{m}\left(j \frac{\widehat{\omega}_{d}}{T}\right)(N-1-d)^{u} e^{-j \widehat{\omega}_{d}(N-1-d)}\right] \\
\boldsymbol{d}_{\hat{\omega}_{d}=} & {\left[c, \boldsymbol{O}_{K_{\hat{\omega}_{d}}-1}^{T}\right]^{\mathrm{T}} . }
\end{aligned}
$$

As linear equality constraints, (13) can readily be incorporated in the SOCP framework. It should be noted that the aforementioned constraints can be imposed at more than one frequency 
point as long as the total number of linear equality constraints does not exceed the length of vector $f$.

2) Convex quadratic inequality constraints: Apart from linear equality constraints, linear and quadratic inequality constraints can easily be incorporated in the SOCP framework. As an illustration, we shall impose a prescribed aliasing-error ripple constraint to meet a given SFDR mentioned in Section II-B. Letting $\delta_{\text {peak }}$ be the desired peak aliasing error to be imposed in a frequency band $\omega \in\left[\omega_{s 1}, \omega_{s 2}\right]$ (a collection of frequency bands is also feasible), the peak error constraint can be written as

$$
\left|T_{p}\left(e^{j \omega}\right)\right| \leq \delta_{\text {peak }}, \quad p=1, \ldots, M-1, \quad \omega \in\left[\omega_{s 1}, \omega_{s 2}\right] .
$$

Similar to the minimax case, (14) can be rewritten as

$$
\delta_{\text {peak }} \geq\left\|\boldsymbol{R}_{p} \boldsymbol{x}\right\|_{2}
$$

where $\boldsymbol{R}_{p}=\left[\begin{array}{cc}0 & \boldsymbol{r}_{p}^{T}(\omega) \\ 0 & \boldsymbol{i}_{p}^{T}(\omega)\end{array}\right]$. Discretizing (15), the resulting constraints on the peak ripples can be augmented to the existing constraints in (7) and (10) for the minimax and LS criteria as a set of second-order cone constraints, respectively.

\section{Multiplierless Realization of the Proposed Digital SYNTHESIS FILTERS}

In this section, the multiplierless realization and word-length determination of the designed digital synthesis filters to meet a given SFDR are described successively in Sections III-A and III-B. Since adder cells and/or registers are usually the major hardware resources in our systems, their numbers are employed as the measure of the hardware complexity. Other measures can also be used with slight modification of these algorithms.

\section{A. Multiplierless Realization Using SOPOT Coefficients}

In the SOPOT representations, the constant coefficients in each digital filter can efficiently be implemented as limited number of shifts and additions as follows:

$$
f_{m}^{\text {sopot }}(n)=\sum_{r=1}^{R} b_{r} 2^{a_{r}}
$$

where $b_{r} \in\{-1,1\}$ and $a_{r} \in\left\{-c_{l}, \ldots,-1,0,1, \ldots, c_{\mathrm{u}}\right\} ; c_{l}$ and $c_{\mathrm{u}}$ are positive integers, and their values determine the dynamic range of the coefficients; and $R$ is the number of terms used in the coefficient approximation. The SOPOT representation for the designed real-valued coefficients $f$ 's of digital synthesis filters can be obtained by a number of existing methods [12], [14], [16]. Here, we shall employ the random-search algorithm reported in [16] because different types of constraints can easily be incorporated. The objective function to be minimized can be written as follows:

$$
\begin{aligned}
\min & \left\langle N_{\text {SOPOT }}\right\rangle \\
\text { subject to } & \begin{cases}\xi_{p}<\xi_{\mathrm{p}-\max }, & p=0 \\
\xi_{p}<\xi_{\mathrm{s}-\max }, & p=1, \ldots, M-1\end{cases}
\end{aligned}
$$

where $\xi_{p}=\max \left\langle\left|T_{p}^{\mathrm{SOPOT}}\left(e^{j \omega}\right)-D_{p}\left(e^{j \omega}\right)\right|\right\rangle$ is the peak ripple error; $N_{\mathrm{SOPOT}}$ is the total number of SOPOT terms used to implement all the SOPOT coefficients; $T_{p}^{\mathrm{SOPOT}}\left(e^{j \omega}\right)$ is the transfer function of the HFB ADC where digital synthesis filters are represented using SOPOT coefficients; $\xi_{\mathrm{p}-\max }$ is the maximum tolerance of the peak reconstruction distortion error; and $\xi_{\mathrm{s}-\max }$ is the maximum tolerance of the peak aliasing error. The selection of $\xi_{\mathrm{p} \text {-max }}$ and $\xi_{\mathrm{s}-\max }$ is related to the desired SFDR, and it will be further discussed in Section IV. Let $f^{\text {SOPOT }}$ be the vector containing the SOPOT coefficients. The random-search algorithm repetitively calculates a candidate $f^{\mathrm{SOPOT}}$ given by

$$
\boldsymbol{f}^{\mathrm{SOPOT}}=\left[\boldsymbol{f}+\lambda \boldsymbol{f}_{b}\right]_{\mathrm{SOPOT}}
$$

where $f_{b}$ is a random vector with elements chosen in the range of $\pm 1, \lambda$ is a user-defined variable used to control the size of the neighborhood to be searched, and $[\cdot]_{\text {SOPOT }}$ is the rounding operator that converts every element inside the input vector to its closest SOPOT value bounded between $-c_{l}$ and $c_{u}$. The set that yields the minimum total number of SOPOT terms, while satisfying the given specifications, is declared as the optimum solution. To further reduce the implementation complexity, the MB technique proposed in [15] is also employed. The MB method reduces the redundancies in implementing all the SOPOT coefficients by removing possible common subexpressions in their representations. FIR synthesis filters can be implemented in their transposed form so that redundant additions in the SOPOT products can be removed by an MB, which greatly reduces the arithmetic complexity. More details of the MB technique can be found in [15].

\section{B. Word-Length Determination}

Once the SOPOT coefficients are obtained, the word length of the intermediate signals shall be determined to minimize the hardware costs while satisfying the given SFDR budget. Here, the word length is allowed to be variable, and the GP approach proposed in [18] is adopted for word-length determination.

In the fixed-point arithmetic, each intermediate signal can be represented in the form of $\langle I / F\rangle$, where $I$ is the number of integer bits and $F$ is the number of fractional bits. We shall minimize the number of fractional bits of the intermediate signals while satisfying the prescribed accuracy. Letting $\sigma_{\text {sum }}^{2}$ be the maximum allowed signal round-off noise power for a given finite word-length accuracy at the channel output, the problem of finding the word lengths of digital synthesis filters can be formulated as the following optimization problem:

$$
\begin{aligned}
\min _{b} \quad u_{0}(\boldsymbol{b}) & =\sum_{i=1}^{K} w_{i} b_{i}=\boldsymbol{w}^{T} \boldsymbol{b} \\
\text { subject to } \quad u_{1}(\boldsymbol{b}) & =\frac{1}{3} \sum_{i=1}^{K} c_{i} 2^{-2 b_{i}}-\sigma_{\text {sum }}^{2} \leq 0
\end{aligned}
$$

where $\boldsymbol{w}$ is a constant weight vector, $\boldsymbol{b}$ is the variable vector representing the fractional part of the word lengths of all rounding sources to be determined, $c_{i}=(1 / 2 \pi) \int_{-\pi}^{\pi}\left|G_{i}\left(e^{j \omega}\right)\right|^{2} \mathrm{~d} \omega=$ $\sum_{k} g_{i}^{2}(k), G_{i}\left(e^{j \omega}\right)$ is the transfer function from the $i$ th 
rounding source to the channel output, and $g_{i}(k)$ is the impulse response corresponding to $G_{i}\left(e^{j \omega}\right)$. In most cases, $w_{i}$, the $i$ th element of $w$, is chosen as one for all $i$ 's. The selection of $\sigma_{\text {sum }}^{2}$ will be discussed later in Section IV. If we allow $\boldsymbol{b}$ to take on real values, and introducing a new variable $x_{i}=2^{2 b_{i}}$, one gets the equivalent GP problem of (19) as follows:

$$
\begin{aligned}
\min _{x} & v_{0}(\boldsymbol{x}) \\
\text { subject to } & v_{1}(\boldsymbol{x}) \leq \sigma_{\text {sum }}^{2} \\
& x_{i} \geq 1, \quad i=1, \ldots, K
\end{aligned}
$$

where $v_{0}(\boldsymbol{x})=\prod_{i=1}^{K}\left(x_{i}\right)^{\left[w_{i} /(2 \ln 2)\right]}$ and $v_{1}(\boldsymbol{x})=$ $(1 / 3) \sum_{i=1}^{K} c_{i} x_{i}^{-1}$. The constraint $x_{i} \geq 1$ is used to avoid a negative value of $b_{i}$. As the solution of $b_{i}$ is real valued, the required integer solution can be obtained by rounding them off to the next largest integers or can further be refined using the bit-allocation algorithm [18].

\section{ERROR ANALYSIS AND SFDR OF HBF ADCS}

From the previous discussion, we can see that, when implementing HFB ADCs, there are four main sources of errors: the quantization noises from $M$ ADCs in the ADC array, the intermediate-signal round-off noises due to their finite word lengths, the coefficient round-off error after using the SOPOT representation, and the aliasing errors caused by the HFB structure. For a single-tone input signal with amplitude $A$, the maximum rms value of the aliasing component will be $2 \xi_{\mathrm{s}-\max } A$ since, in the worse case, two aliasing components may overlap with each other. After using the SOPOT representation, the minimum value of the signal amplitude is $A\left(1-\xi_{\mathrm{p}-\max }\right)$. The signal round-off error and ADC quantization error will further yield a component with an rms value that is equal to $\sigma=\sqrt{\sigma_{\text {sum }}^{2}+\sigma_{\text {adc }}^{2}}$ since the two components can be modeled as independent white Gaussian processes. $\sigma_{\text {adc }}^{2}$ is the noise power at the output of the HFB ADC due to the quantization error of $M \mathrm{ADCs}$ in the subchannels. As a result, the SFDR at the channel output is

$$
\mathrm{SFDR}_{\mathrm{HFB}-\mathrm{ADC}}=\frac{A\left(1-\xi_{p-\max }\right) / \sqrt{2}}{\sqrt{2 \xi_{s-\max }^{2} A^{2}+\sigma_{\mathrm{adc}}^{2}+\sigma_{\mathrm{sum}}^{2}}} .
$$

We now evaluate $\sigma_{\text {adc }}^{2}$. For sufficiently large number of bits, the quantization error $e_{i}(n)$ of the ADC in the $i$ th subchannel can be modeled as a random process with zero mean and variance $\sigma_{q}^{2}=2^{-2 B} / 3$, where $B$ is the number of bits in each ADC. The noise component due to the $i$ th branch is

$$
\eta_{i}(n)=\sum_{k=-\infty}^{+\infty} f_{i}(n-M k) e_{i}(k)
$$

where $f_{i}(n)$ is the impulse response of the $i$ th synthesis filter. $\eta_{i}(n)$ is cyclostationary with zero mean and variances

$$
\begin{aligned}
\sigma_{\eta_{i}}^{2} & =\sum_{k=-\infty}^{\infty} \sum_{l=-\infty}^{\infty} f_{i}(n-M k) f_{i}(n-M l) E\left[e_{i}(k) e_{i}(l)\right] \\
& =\sum_{k=-\infty}^{\infty} \sum_{l=-\infty}^{\infty} f_{i}(n-M k) f_{i}(n-M l) \sigma_{q}^{2} \delta(k-l) \\
& =\sigma_{q}^{2} \sum_{k=-\infty}^{\infty} f_{i, n}^{2}(k)
\end{aligned}
$$

where $f_{i, n}(k)$ is the impulse response of the $n$th polyphase component of $f_{i}(k)$. Hence, $\sigma_{\eta_{i}}^{2}$ is periodic with a period of $M$. Since the quantization noises of ADCs can be considered to be independent, the total output power is given by

$$
\sigma_{\text {adc }}^{2}(n)=\sum_{i=1}^{M} \sigma_{\eta_{i}}^{2}(n)=\sigma_{q}^{2} \sum_{i=1}^{M} \sum_{k=-\infty}^{+\infty} f_{i, n}^{2}(k) .
$$

Letting $h_{\max }^{2}=\max _{n} \sum_{i=1}^{M} \sum_{k=-\infty}^{+\infty} f_{i, n}^{2}(k)$, we have

$$
\sigma_{\text {adc }}^{2}(n) \leq \sigma_{\mathrm{q}}^{2} h_{\max }^{2}
$$

$$
S F D R_{\mathrm{HFB}-\mathrm{ADC}} \geq S F D R_{L}
$$

where

$$
\begin{aligned}
S F D R_{L} & =\frac{A\left(1-\xi_{p-\max }\right) / \sqrt{2}}{\sqrt{2 \xi_{s-\max }^{2} A^{2}+\sigma_{\max }^{2}}} \\
\sigma_{\max } & =\sqrt{\sigma_{q}^{2} h_{\max }^{2}+\sigma_{\text {sum }}^{2}} .
\end{aligned}
$$

The effective number of bits of this ADC is then given by

$$
\begin{aligned}
& S F D R_{\mathrm{HFB}-\mathrm{ADC}-\mathrm{bits}} \\
& =S F D R_{\mathrm{HFB}-\mathrm{ADC}}(\mathrm{dBc}) / 6.02 \\
& \geq 20\left\{\log _{10} A\left(1-\xi_{p \text {-max }}\right)\right. \\
& \left.\quad \quad-\log _{10}\left(\sqrt{4 \xi_{s-\max }^{2} A^{2}+2 \sigma_{\max }^{2}}\right)\right\} / 6.02 .
\end{aligned}
$$

For the unit signal amplitude, one gets

$$
\begin{aligned}
& S F D R_{\text {HFB-ADC-bits }} \\
& \quad \geq 20\left\{\log _{10}\left(1-\xi_{p \text {-max }}-\log _{10}\left(\sqrt{4 \xi_{s-\max }^{2}+2 \sigma_{\max }^{2}}\right)\right\} / 6.02 .\right.
\end{aligned}
$$

Therefore, to satisfy the specified reconstruction accuracy in terms of SFDR or effective number of bits, $S F D R_{L}$ should be equal or larger than $S F D R_{d}$ so that $S F D R_{\mathrm{HFB}-\mathrm{ADC}} \geq$ $S F D R_{d}$, where $S F D R_{d}$ is the desired SFDR.

\section{DESIGN With PARAMETER UnCERTAINTIES}

Another objective of this paper is to study the imperfections of HFB ADCs and their effects on the reconstruction results. There are two main imperfections in HFB ADCs: the mismatch errors, such as gain and time mismatch between converters, and the component variations of analog analysis filters. The robustness of HBF ADCs to the mismatch errors has been illustrated in [3]. Therefore, in this paper, we only focus on the component uncertainties in analog analysis filters. 
Hardware components, such as transistors and resistors of analog analysis filters, tend to be influenced by environments and manufacturing processes. Hence, the characteristics of analog analysis filters may be influenced by these parameter uncertainties. Assuming that there are $L$ such analog parameters $l_{m, i}$ in the $m$ th analysis filter that could possibly be affected with uncertainties $\Delta l_{m, i}$, the frequency response of the $m$ th analog analysis filter can be modeled by the first-order Taylor series expansion as follows:

$$
H_{m}(j \Omega)=\bar{H}_{m}(j \Omega)+\boldsymbol{\Phi}_{m}^{T}(j \Omega) \Delta \boldsymbol{l}_{m}
$$

where

$\boldsymbol{\Phi}_{m}(j \Omega)=\left[\partial H_{m}(j \Omega) / \partial l_{m, 0}, \ldots, \partial H_{m}(j \Omega) / \partial l_{m, L-1}\right]^{\mathrm{T}}$, $\Delta l_{m, i}$ is the variation of the $i$ th parameter of the $m$ th analog analysis filter, $\Delta \boldsymbol{l}_{m}=\left[\Delta l_{m, 0}, \ldots, \Delta l_{m, L-1}\right]^{\mathrm{T}}$, and $\bar{H}_{m}(j \Omega)$ is the desired frequency response of the $m$ th analog analysis filter. Accordingly, the transfer function $T_{p}\left(e^{j \omega}\right)$ can be written as

$$
\begin{array}{r}
T_{p}\left(e^{j \omega}\right)=\frac{1}{M T} \sum_{m=0}^{M-1} F_{m}\left(e^{j \omega}\right)\left[\bar{H}_{m}\left(j \frac{\omega}{T}-j \frac{2 \pi p}{M T}\right)\right. \\
\left.+\Delta H_{m}\left(j \frac{\omega}{T}-j \frac{2 \pi p}{M T}\right)\right]
\end{array}
$$

where $\Delta H_{m}(j(\omega / T))=\boldsymbol{\Phi}_{m}^{T}(j(\omega / T)) \Delta \boldsymbol{l}_{m}$. Substituting (29) into the approximation error $e_{p}(\omega)$ in (4), we have

$$
\hat{e}_{p}(\omega)=\bar{T}_{p}\left(e^{j \omega}\right)-D_{p}\left(e^{j \omega}\right)+\Delta T_{p}\left(e^{j \omega}\right)
$$

where

$$
\begin{aligned}
\bar{T}_{p}\left(e^{j \omega}\right) & =\frac{1}{M T} \sum_{m=0}^{M-1} F_{m}\left(e^{j \omega}\right) \bar{H}_{m}\left(j \frac{\omega}{T}-j \frac{2 \pi p}{M T}\right) \\
\Delta T_{p}\left(e^{j \omega}\right) & =\frac{1}{M T} \sum_{m=0}^{M-1} F_{m}\left(e^{j \omega}\right) \Delta H_{m}\left(j \frac{\omega}{T}-j \frac{2 \pi p}{M T}\right) .
\end{aligned}
$$

Compared to (4), (30) has an additional term $\Delta T_{p}\left(e^{j \omega}\right)$, which originates from the parameter variations. If only the first two terms are considered, (30) will reduce to the nominal-design algorithm discussed previously. The reconstruction result may seriously be degraded due to $\Delta T_{p}\left(e^{j \omega}\right)$. To reduce the sensitivity and effects of parameter variations on the reconstruction quality, two robust design methods, namely, the SRD and WCRD, are proposed hereinafter.

\section{A. Stochastic Robust Design (SRD)}

In SRD, $\Delta l_{m, i}$ 's are treated as zero-mean uncorrelated random variables with variance $\sigma_{m, i}^{2}$. For simplicity, $\sigma_{m, i}^{2}$ is assumed to be identical for all $i$ 's and is denoted by $\sigma_{m}^{2}$. The weighted peak expected value of $\left|\hat{e}_{p}(\omega)\right|$ is minimized and leads to the following problem:

$$
\min _{\boldsymbol{f}}\left\{\max _{p, \omega} E\left[W_{p}(\omega)\left|\hat{e}_{p}(\omega)\right|\right]\right\}
$$

where $E[\cdot]$ denotes the expectation operator. More specifically, $\left|\hat{e}_{p}(\omega)\right|$ can be expressed as follows:

$$
\begin{aligned}
\left|\hat{e}_{p}(\omega)\right|= & \left|\bar{T}_{p}\left(e^{j \omega}\right)-D_{p}\left(e^{j \omega}\right)+\Delta T_{p}\left(e^{j \omega}\right)\right| \\
= & \mid \frac{1}{M T} \sum_{m=0}^{M-1} F_{m}\left(e^{j \omega}\right) \bar{H}_{m}\left(j \frac{\omega}{T}-j \frac{2 \pi p}{M T}\right)-D_{p}\left(e^{j \omega}\right) \\
& +\frac{1}{M T} \sum_{m=0}^{M-1} F_{m}\left(e^{j \omega}\right) \Delta H_{m}\left(j \frac{\omega}{T}-j \frac{2 \pi p}{M T}\right) \mid .
\end{aligned}
$$

To simplify the notation, we define

$$
\begin{aligned}
\overline{\boldsymbol{r}}_{p, m}(\omega) & =\left[\frac{1}{M T} \bar{H}_{m}\left(j \frac{\omega}{T}-j \frac{2 \pi p}{M T}\right)(\boldsymbol{c}(\omega)-j \boldsymbol{s}(\omega))\right]_{R} \\
\overline{\boldsymbol{i}}_{p, m}(\omega) & =\left[\frac{1}{M T} \bar{H}_{m}\left(j \frac{\omega}{T}-j \frac{2 \pi p}{M T}\right)(\boldsymbol{c}(\omega)-j \boldsymbol{s}(\omega))\right]_{I} \\
\boldsymbol{r}_{p . m}(\omega) & =\left[\frac{1}{M T} \Delta H_{m}\left(j \frac{\omega}{T}-j \frac{2 \pi p}{M T}\right)(\boldsymbol{c}(\omega)-j \boldsymbol{s}(\omega))\right]_{R} \\
\hat{\boldsymbol{i}}_{p \cdot m}(\omega) & =\left[\frac{1}{M T} \Delta H_{m}\left(j \frac{\omega}{T}-j \frac{2 \pi p}{M T}\right)(\boldsymbol{c}(\omega)-j \boldsymbol{s}(\omega))\right]_{I} .
\end{aligned}
$$

Thus, $\left|\hat{e}_{p}(\omega)\right|$ can be written in a more compact matrix form as

$$
\left|\hat{e}_{p}(\omega)\right|=\left\|\left(\overline{\boldsymbol{A}}_{p}(\omega)+\widehat{\boldsymbol{A}}_{p}(\omega)\right) \boldsymbol{f}-\boldsymbol{b}_{p}(\omega)\right\|_{2}
$$

where

$$
\begin{aligned}
\boldsymbol{b}_{p}(\omega) & =\left[R_{p}^{\mathrm{d}}(\omega), I_{p}^{\mathrm{d}}(\omega)\right]^{\mathrm{T}} \\
\overline{\boldsymbol{A}}_{p}(\omega) & =\left[\overline{\boldsymbol{r}}_{p}(\omega), \overline{\boldsymbol{i}}_{p}(\omega)\right]^{\mathrm{T}} \\
\widehat{\boldsymbol{A}}_{p}(\omega) & =\left[\hat{\boldsymbol{r}}_{p}(\omega), \widehat{\boldsymbol{i}}_{p}(\omega)\right]^{\mathrm{T}} \\
\overline{\boldsymbol{r}}_{p}(\omega) & =\left[\overline{\boldsymbol{r}}_{p, 0}^{T}(\omega), \ldots, \overline{\boldsymbol{r}}_{p, M-1}^{T}(\omega)\right]^{\mathrm{T}} \\
\widehat{\boldsymbol{r}}_{p}(\omega) & =\left[\hat{\boldsymbol{r}}_{p, 0}^{T}(\omega), \ldots, \widehat{\boldsymbol{r}}_{p, M-1}^{T}(\omega)\right]^{\mathrm{T}} \\
\overline{\boldsymbol{i}}_{p}(\omega) & =\left[\overline{\boldsymbol{i}}_{p, 0}^{T}(\omega), \ldots, \overline{\boldsymbol{i}}_{p, M-1}^{T}(\omega)\right]^{\mathrm{T}} \\
\widehat{\boldsymbol{i}}_{p}(\omega) & =\left[\hat{\boldsymbol{i}}_{p, 0}^{T}(\omega), \ldots, \widehat{\boldsymbol{i}}_{p, M-1}^{T}(\omega)\right]^{\mathrm{T}} .
\end{aligned}
$$

Taking the expectation of $W_{p}(\omega)\left|\hat{e}_{p}(\omega)\right|$, we have

$$
\begin{aligned}
E & {\left[W_{p}(\omega)\left|\hat{e}_{p}(\omega)\right|\right] } \\
& =E\left[W_{p}(\omega)\left\|\left(\overline{\boldsymbol{A}}_{p}(\omega)+\widehat{\boldsymbol{A}}_{p}(\omega)\right) \boldsymbol{f}-\boldsymbol{b}_{p}(\omega)\right\|_{2}\right] \\
& =W_{p}(\omega)\left[\left\|\overline{\boldsymbol{A}}_{p}(\omega) \boldsymbol{f}-\boldsymbol{b}_{p}(\omega)\right\|_{2}^{2}+\left\|\boldsymbol{P}_{p}^{1 / 2}(\omega) \boldsymbol{f}\right\|_{2}^{2}\right]^{1 / 2} \\
& =\left\|\boldsymbol{A}_{p}(\omega) \boldsymbol{f}-\boldsymbol{d}_{p}(\omega)\right\|_{2}
\end{aligned}
$$

where $\boldsymbol{A}_{p}(\omega)=W_{p}(\omega)\left[\overline{\boldsymbol{A}}_{p}^{T}(\omega), \boldsymbol{P}_{p}^{1 / 2}(\omega)\right]^{\mathrm{T}}, \boldsymbol{d}_{p}(\omega)=$ $W_{p}(\omega)\left[\boldsymbol{b}_{p}^{T}(\omega), \boldsymbol{O}_{N M}^{T}\right]^{\mathrm{T}}$, and $\boldsymbol{P}_{p}(\omega)=E\left[\widehat{\boldsymbol{A}}_{p}^{T}(\omega) \widehat{\boldsymbol{A}}_{p}(\omega)\right]$. After some manipulations, $\boldsymbol{P}_{p}(\omega)$ becomes a block-diagonal matrix, with the $(m, m)$ th $(N \times N)$ block given by

$\frac{1}{(M T)^{2}}\left\|\boldsymbol{\Phi}_{m}\left(j \frac{\omega}{T}\right)\right\|_{2}^{2} \sigma_{m}^{2}[\boldsymbol{c}(\omega), \boldsymbol{s}(\omega)][\boldsymbol{c}(\omega), \boldsymbol{s}(\omega)]^{\mathrm{T}}$, $m=1, \ldots, M$ 
Hence, the design problem is formulated as the following SOCP problem:

$$
\begin{array}{cl}
\min _{f} & \delta \\
\text { subject to } & \delta \geq\left\|\boldsymbol{A}_{p}(\omega) \boldsymbol{f}-\boldsymbol{d}_{p}(\omega)\right\|_{2} .
\end{array}
$$

Discretizing the frequency variable $\omega$ over a dense set of frequencies $\left\{\omega_{i}, 1 \leq i \leq K_{\mathrm{s}}\right\}$ on the frequency band of interest, the constraints become $\delta \geq\left\|\boldsymbol{A}_{p}\left(\omega_{i}\right) \boldsymbol{f}-\boldsymbol{d}_{p}\left(\omega_{i}\right)\right\|_{2}$. Then, (34) can be written in a standard SOCP form as follows:

$$
\min _{x_{\mathrm{srd}}} \boldsymbol{b}_{\mathrm{srd}}^{T} \boldsymbol{x}_{\mathrm{srd}}
$$$$
\text { subject to } \boldsymbol{b}_{\mathrm{srd}}^{T} \boldsymbol{x}_{\mathrm{srd}} \geq\left\|\boldsymbol{F}_{p, i}^{\mathrm{srd}} \boldsymbol{x}_{\mathrm{srd}}-\boldsymbol{d}_{p, i}\right\|_{2}
$$

where $\boldsymbol{b}_{\text {srd }}=\left[1, \boldsymbol{O}_{N M}^{T}\right]^{\mathrm{T}}, \boldsymbol{x}_{\text {srd }}=\left[\delta, \boldsymbol{f}^{T}\right]^{\mathrm{T}}, \boldsymbol{F}_{p, i}^{\mathrm{srd}}=$ $\left[\boldsymbol{O}_{N M+2}, \boldsymbol{A}_{p}\left(\omega_{i}\right)\right], \boldsymbol{d}_{p, i}=\boldsymbol{d}_{p}\left(\omega_{i}\right), p=0, \ldots, M-1$, and $1 \leq i \leq K_{s}$. Equation (35) can readily be solved using a standard SOCP solver.

\section{B. WCRD}

Instead of minimizing the weighted peak expected value of $\left|\hat{e}_{p}(\omega)\right|$, we can minimize the weighted worst-case error $\hat{e}_{\mathrm{wc}}(\omega)$ for bounded uncertainties in the variations, i.e., $\left\|\Delta \boldsymbol{l}_{m}\right\|_{2} \leq \tau$, where $\hat{e}_{\mathrm{wc}}(\omega)=\sup \left[W_{p}(\omega)\left|\hat{e}_{p}(\omega)\right|\right], \sup [x]$ returns the maximum value of $x$, and $\tau$ is a bound obtained from prior knowledge on the parameter variations. This is referred to as the WCRD. First of all, rewrite $\Delta T_{p}\left(e^{j w}\right)$ as

$$
\begin{aligned}
\Delta & T_{p}\left(e^{j \omega}\right) \\
& =\frac{1}{M T} \sum_{m=0}^{M-1} F_{m}\left(e^{j \omega}\right) \Delta H_{m}\left(j \frac{\omega}{T}-j \frac{2 \pi p}{M T}\right) \\
& =\frac{1}{M T} \sum_{m=0}^{M-1} \boldsymbol{f}_{m}^{T}(\boldsymbol{c}(\omega)-j \boldsymbol{s}(\omega)) \boldsymbol{\Phi}_{m}^{T}\left(j \frac{\omega}{T}-j \frac{2 \pi p}{M}\right) \Delta \boldsymbol{l}_{m} \\
& =\left\{\boldsymbol{\Delta} \boldsymbol{l}^{T} \cdot \boldsymbol{P}_{-} \boldsymbol{C}_{p}^{T}(\omega)+j \boldsymbol{\Delta} \boldsymbol{l}^{T} \cdot \boldsymbol{P}_{-} \boldsymbol{S}_{p}^{T}(\omega)\right\} \boldsymbol{f}
\end{aligned}
$$

where

$$
\begin{aligned}
& \boldsymbol{P}_{-} \boldsymbol{C}_{p}(\omega)=\left[\boldsymbol{P}_{-} \boldsymbol{C}_{p, 0}^{T}(\omega), \ldots, \boldsymbol{P}_{-} \boldsymbol{C}_{p, M-1}^{T}(\omega)\right]^{\mathrm{T}} \\
& \boldsymbol{P}_{\boldsymbol{S}_{p}}(\omega)=\left[\boldsymbol{P} \boldsymbol{S}_{p, 0}^{T}(\omega), \ldots, \boldsymbol{P}_{-} \boldsymbol{S}_{p, M-1}^{T}(\omega)\right]^{\mathrm{T}} \\
& \boldsymbol{P}_{-} \boldsymbol{C}_{p, m}(\omega)=\left[\frac{1}{M T}(\boldsymbol{c}(\omega)-j \boldsymbol{s}(\omega)) \widehat{\boldsymbol{\Phi}}_{m}^{T}\left(j \frac{\omega}{T}-j \frac{2 \pi p}{M T}\right)\right]_{R} \\
& P_{-} \boldsymbol{S}_{p, m}(\omega)=\left[\frac{1}{M T}(\boldsymbol{c}(\omega)-j \boldsymbol{s}(\omega)) \widehat{\boldsymbol{\Phi}}_{m}^{T}\left(j \omega T-j \frac{2 \pi p}{M T}\right)\right]_{I} \\
& \Delta l=\left[\Delta \boldsymbol{l}_{0}^{T}, \ldots, \Delta \boldsymbol{l}_{M-1}^{T}\right]^{\mathrm{T}} \\
& \widehat{\boldsymbol{\Phi}}_{m}=\left[\boldsymbol{O}_{m L}^{T}, \boldsymbol{\Phi}_{m}^{T}, \boldsymbol{O}_{(M-m-1) L}^{T}\right]^{\mathrm{T}} \text {. }
\end{aligned}
$$

Substituting (36) into (30), we have

$$
\begin{aligned}
\left|\hat{e}_{p}(\omega)\right| & =\left|\bar{T}_{p}\left(e^{j \omega}\right)-D_{p}\left(e^{j \omega}\right)+\Delta T_{p}\left(e^{j \omega}\right)\right| \\
& =\left\|\left[\begin{array}{c}
\bar{r}_{p}^{T}(\omega)+\Delta \boldsymbol{l}^{T} \cdot \boldsymbol{P}_{-} \boldsymbol{C}_{p}^{T}(\omega) \\
\overline{\boldsymbol{i}}_{p}^{T}(\omega)+\Delta \boldsymbol{l}^{T} \cdot \boldsymbol{P}_{-} \boldsymbol{S}_{p}^{T}(\omega)
\end{array}\right] \boldsymbol{f}-\boldsymbol{b}_{p}(\omega)\right\|_{2} .
\end{aligned}
$$

To minimize the weighted worst case error of a candidate solution, the resultant design problem can be formulated as

$$
\min _{f}\left\{\max _{p, \omega} W_{p}(\omega)\left|\hat{e}_{p}(\omega)\right|\right\}
$$

Since

$$
\begin{aligned}
& \sup \left[\left|\overline{\boldsymbol{r}}_{p}^{T}(\omega) \boldsymbol{f}+\Delta \boldsymbol{l}^{T} \cdot \boldsymbol{P} \_C_{p}^{T}(\omega) \boldsymbol{f}-R_{p}^{\mathrm{d}}(\omega)\right|\right] \\
& \quad \leq\left|\overline{\boldsymbol{r}}_{p}^{T}(\omega) \boldsymbol{f}-R_{p}^{\mathrm{d}}(\omega)\right|+\left|\Delta \boldsymbol{l}^{T} \cdot \boldsymbol{P} \_\boldsymbol{C}_{p}^{T} \boldsymbol{f}\right| \\
& \quad \leq\left|\overline{\boldsymbol{r}}_{p}^{T}(\omega) \boldsymbol{f}-R_{p}^{\mathrm{d}}(\omega)\right|+\|\Delta \boldsymbol{l}\|_{2}\left\|\boldsymbol{P} \boldsymbol{C}_{p}^{T} \boldsymbol{f}\right\|_{2}
\end{aligned}
$$

and $\left\|\Delta \boldsymbol{l}_{m}\right\|_{2} \leq \tau$, we have

$$
\begin{aligned}
\sup \left[\mid \overline{\boldsymbol{r}}_{p}^{T}(\omega) \boldsymbol{f}+\right. & \left.\boldsymbol{P}_{-} \boldsymbol{C}_{p}^{T}(\omega) \boldsymbol{f}-R_{p}^{\mathrm{d}}(\omega) \mid\right] \\
& \leq\left|\overline{\boldsymbol{r}}_{p}^{T}(\omega) \boldsymbol{f}-R_{p}^{\mathrm{d}}(\omega)\right|+M \tau\left\|\boldsymbol{P}_{-} \boldsymbol{C}_{p}^{T}(\omega) \boldsymbol{f}\right\|_{2} .
\end{aligned}
$$

Accordingly, we have

$$
\begin{aligned}
\sup \left[\left|\overline{\boldsymbol{i}}_{p}^{T}(\omega) \boldsymbol{f}+\boldsymbol{P}_{-} \boldsymbol{S}_{p}^{T}(\omega) \boldsymbol{f}-I_{p}^{\mathrm{d}}(\omega)\right|\right] \\
\leq\left|\overline{\boldsymbol{i}}_{p}^{T}(\omega) \boldsymbol{f}-I_{p}^{\mathrm{d}}(\omega)\right|+M \tau\left\|\boldsymbol{P} \boldsymbol{S}_{p}^{T}(\omega) \boldsymbol{f}\right\|_{2} .
\end{aligned}
$$

Hence, to minimize the weighted worst case error, the problem in (38) is reformulated as

$$
\min _{\boldsymbol{f}}\|\boldsymbol{t}\|_{2}
$$

subject to $t_{1} \geq W_{p}(\omega)\left[\left|\overline{\boldsymbol{r}}_{p}^{T}(\omega) \boldsymbol{f}-R_{p}^{\mathrm{d}}(\omega)\right|\right.$

$$
\begin{array}{r}
\left.+M \tau\left\|\boldsymbol{P} \_C_{p}^{T}(\omega) \boldsymbol{f}\right\|_{2}\right] \\
t_{2} \geq W_{p}(\omega)\left[\left|\overline{\boldsymbol{i}}_{p}^{T}(\omega) \boldsymbol{f}-I_{p}^{\mathrm{d}}(\omega)\right|\right. \\
\left.+M \tau\left\|\boldsymbol{P} \boldsymbol{S}_{p}^{T}(\omega) \boldsymbol{f}\right\|_{2}\right]
\end{array}
$$

where $\boldsymbol{t}=\left[\begin{array}{ll}t_{1} & t_{2}\end{array}\right]^{\mathrm{T}}$. This problem can be formulated as $\min _{f} \delta$

subject to $\delta \geq\|\boldsymbol{t}\|_{2}$

$$
\begin{aligned}
& t_{1}+W_{p}(\omega)\left[\overline{\boldsymbol{r}}_{p}^{T}(\omega) \boldsymbol{f}-R_{p}^{\mathrm{d}}(\omega)\right] \geq\left\|M \tau W_{p}(\omega) \boldsymbol{P} \_\boldsymbol{C}_{p}^{T}(\omega) \boldsymbol{f}\right\|_{2} \\
& t_{1}-W_{p}(\omega)\left[\overline{\boldsymbol{r}}_{p}^{T}(\omega) \boldsymbol{f}-R_{p}^{\mathrm{d}}(\omega)\right] \geq\left\|M \tau W_{p}(\omega) \boldsymbol{P} \boldsymbol{C}_{p}^{T}(\omega) \boldsymbol{f}\right\|_{2} \\
& t_{2}+W_{p}(\omega)\left[\overline{\boldsymbol{i}}_{p}^{T}(\omega) \boldsymbol{f}-I_{p}^{\mathrm{d}}(\omega)\right] \geq\left\|M \tau W_{p}(\omega) \boldsymbol{P} \boldsymbol{S}_{p}^{T}(\omega) \boldsymbol{f}\right\|_{2} \\
& t_{2}-W_{p}(\omega)\left[\overline{\boldsymbol{i}}_{p}^{T}(\omega) \boldsymbol{f}-I_{p}^{\mathrm{d}}(\omega)\right] \geq\left\|M \tau W_{p}(\omega) \boldsymbol{P} \boldsymbol{S}_{p}^{T}(\omega) \boldsymbol{f}\right\|_{2} .
\end{aligned}
$$

Letting $\boldsymbol{x}_{\mathrm{wc}}=\left[\delta, \boldsymbol{t}^{T}, \boldsymbol{f}^{T}\right]^{\mathrm{T}}$ and $\boldsymbol{b}_{\mathrm{wc}}=\left[-1, \boldsymbol{O}_{2+N M}\right]^{\mathrm{T}}$, (39) can be formulated as the following standard SOCP problem:

subject to

$$
\min _{x_{\mathrm{wc}}} \quad \boldsymbol{b}_{\mathrm{wc}}^{T} \boldsymbol{x}_{\mathrm{wc}}
$$

$$
\begin{aligned}
\boldsymbol{c}_{0}^{T} \boldsymbol{x}_{\mathrm{wc}} & \geq\left\|A_{0} \boldsymbol{x}_{\mathrm{wc}}\right\|_{2} \\
\boldsymbol{c}_{1}^{T} \boldsymbol{x}_{\mathrm{wc}}-d_{\mathrm{r}} & \geq\left\|\boldsymbol{A}_{\mathrm{r}} \boldsymbol{x}_{\mathrm{wc}}\right\|_{2} \\
\boldsymbol{c}_{2}^{T} \boldsymbol{x}_{\mathrm{wc}}+d_{\mathrm{r}} & \geq\left\|\boldsymbol{A}_{\mathrm{r}} \boldsymbol{x}_{\mathrm{wc}}\right\|_{2} \\
\boldsymbol{c}_{3}^{T} \boldsymbol{x}_{\mathrm{wc}}-d_{\mathrm{i}} & \geq\left\|\boldsymbol{A}_{\mathrm{i}} \boldsymbol{x}_{\mathrm{wc}}\right\|_{2} \\
\boldsymbol{c}_{4}^{T} \boldsymbol{x}_{\mathrm{wc}}+d_{\mathrm{i}} & \geq\left\|\boldsymbol{A}_{\mathrm{i}} \boldsymbol{x}_{\mathrm{wc}}\right\|_{2}
\end{aligned}
$$


where

$$
\begin{aligned}
d_{\mathrm{r}} & =W_{p}(\omega) R_{p}^{\mathrm{d}}(\omega) \\
d_{\mathrm{i}} & =W_{p}(\omega) I_{p}^{\mathrm{d}}(\omega) \\
\boldsymbol{Z}_{o} & =\left[\boldsymbol{O}_{L M}, \boldsymbol{O}_{L M}, \boldsymbol{O}_{L M}\right] \\
\boldsymbol{A}_{0} & =\left[0,1,0, \boldsymbol{O}_{N M}^{T}\right. \\
\boldsymbol{A}_{\mathrm{r}} & =\left[0,0,1, \boldsymbol{O}_{N M}^{T}\right] \\
\boldsymbol{A}_{\mathrm{i}} & =\left[\boldsymbol{Z}_{o}, M \tau W_{p}(\omega) W_{p}(\omega) \boldsymbol{P}_{-} \boldsymbol{S}_{p}^{T}(\omega)\right] \\
\boldsymbol{c}_{0} & =\left[1, \boldsymbol{O}_{2+N M}^{T}\right] \\
\boldsymbol{c}_{1} & =\left[0,1,0, W_{p}(\omega) \overline{\boldsymbol{r}}_{p}^{T}(\omega)\right]^{\mathrm{T}} \\
\boldsymbol{c}_{2} & =\left[0,1,0,-W_{p}(\omega) \overline{\boldsymbol{r}}_{p}^{T}(\omega)\right]^{\mathrm{T}} \\
\boldsymbol{c}_{3} & =\left[0,0,1, W_{p}(\omega) \overline{\boldsymbol{s}}_{p}^{T}(\omega)\right]^{\mathrm{T}} \\
\boldsymbol{c}_{4} & =\left[0,0,1,-W_{p}(\omega) \overline{\boldsymbol{s}}_{p}^{T}(\omega)\right]^{\mathrm{T}} \\
p & =0, \ldots, M-1 .
\end{aligned}
$$

\section{DESIGN EXAMPLES AND RESUlTS}

In this section, several HFB ADCs are designed to evaluate the effectiveness of the proposed algorithms. The minimax and LS designs, the constrained design with prescribed magnitude flatness and peak aliasing errors, and the robust design with component uncertainties are all considered. Unless otherwise specified, the order of FIR synthesis filters, scale factor $c$, system delay $d$, and the number of frequency sample points of the design examples are set to $80,1,40$, and 100, respectively. The SOCP problems are solved by the SeDuMi Matlab toolbox [20], and GP optimization is carried out using the MOSEK Matlab toolbox [21].

Example 1: Nominal design without parameter uncertainties and constraints: In this example, a four-channel 12-bit system is designed. The desired SFDR is $12 \times 6.02=72.3 \mathrm{~dB}$. As described in Section II, when designing real-valued digital synthesis filters, the aliasing errors should be canceled by a few decibels lower than the desired SFDR to account for the quantization errors in ADCs in the ADC array and the finite wordlength effect during implementation. Then, the aliasing errors are required to be canceled below $-90 \mathrm{~dB}$ in this example. The nominal minimax and LS designs without constraints and parameter uncertainties are considered. Analog analysis filters are chosen as second-order Butterworth filters. FIR synthesis filters are designed using the minimax and LS criteria, as discussed in Section II-B. An oversampling ratio of $6 \%$ is applied so that the input signal is bandlimited to $[0,0.94 \pi]$, and its spectrum is zero at the don't care band $[0.94 \pi, \pi] . W_{p}(\omega)$ for $p=0, \ldots, M-1$ are set to 1 in signal band $[0,0.94 \pi]$ and to 0 at $0.94 \pi, \pi]$. As the error minimization method proposed in [5] also used analog analysis filters with simple structure, this method is also carried out for comparison. The frequency responses of the three designed HFB ADCs are shown in Fig. 2. For the distortion error, the maximum deviations from $0 \mathrm{~dB}$ of the proposed minimax and LS design in the frequency band $[0,0.94 \pi]$ are 0.06 and $0.065 \mathrm{~dB}$, respectively. The aliasing
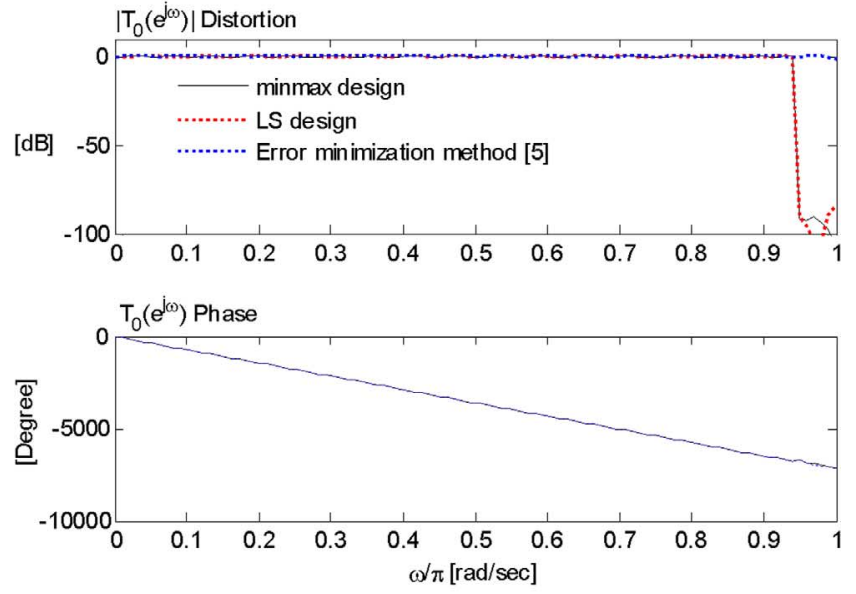

(a)
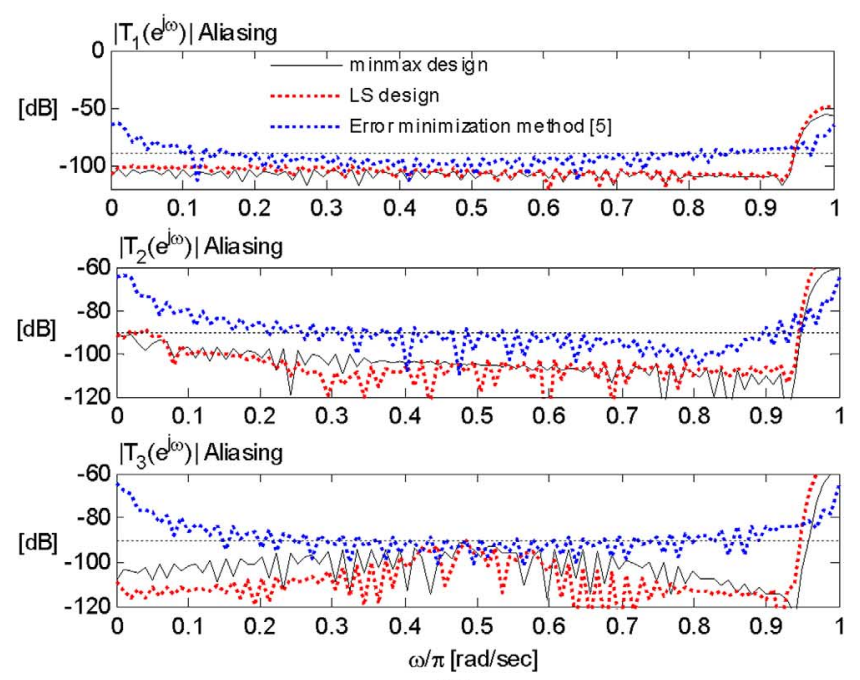

(b)

Fig. 2. Nominal design results in Example 1. (a) Distortion. (b) Aliasing errors.

errors of the two proposed design methods are suppressed to below $-90 \mathrm{~dB}$ in $\omega \in[0,0.94 \pi]$. In contrast, the error minimization method in [5] has a much larger aliasing error in the frequency band $[0,0.2 \pi]$ and cannot satisfy the desired SFDR. The distortion and aliasing errors of the two proposed design methods mostly concentrate at $\omega=\pi$ and do not severely limit the usable bandwidth of the system. This is attributed to the optimality of the SOCP formulation.

We now present the multiplierless realization of synthesis filters of the proposed minimax and LS approaches. Given the coefficients of synthesis filters, the output power of the quantization noises of ADCs can be determined using (24). Some 15-bit ADCs are assumed to be used in the ADC array. The quantization noise power of a 15 -bit ADC is about $3.1 \times 10^{-10}$, and according to (24), we have the quantization noise power at the output given by $\sigma_{\text {adc }}^{2} \leq 6.26 \times 10^{-9}$ for the minimax approach and $\sigma_{\text {adc }}^{2} \leq 6.27 \times 10^{-9}$ for the LS approach. $\xi_{p \text {-max }}$ and $\xi_{s \text {-max }}$ are set to $-40 \mathrm{~dB}$ (the maximum allowed deviation of the magnitude of the reconstructed signal from $0 \mathrm{~dB}$ is $0.09 \mathrm{~dB}$ ) and $-83 \mathrm{~dB}\left(7.08 \times 10^{-5}\right)$, respectively, and $\sigma_{\text {sum }}^{2}$ is required to be smaller than $1.1 \times 10^{-8}$. According to (25), if all specifications are satisfied, we have $S F D R_{\mathrm{HFB}-\mathrm{ADC}} \geq 72.5 \mathrm{~dB}$ for 
the two proposed approaches. Given the accuracy specification, the SOPOT representation for each coefficient of the designed synthesis filters are then determined, as described in Section III. Using the GP formulation, the optimal word-length format for each intermediate signal is obtained. Fig. 3 shows the frequency responses of the two proposed approaches using SOPOT coefficients. In the interested frequency band $[0.94 \pi, \pi]$, for both two approaches, the maximum deviation of the reconstructed signals from $0 \mathrm{~dB}$ is $0.09 \mathrm{~dB}$, and the peak aliasing error in each subchannel is $-83 \mathrm{~dB}$. The output-signal round-off noise is $0.63 \times 10^{-8}$. Hence, the two reconstruction systems have an SFDR that is larger than $73.3 \mathrm{~dB}$, and we have the effective bits $S F D R_{\text {HFB-ADC-bits }} \geq 12.17$ in the interested band. The hardware complexity for the designed synthesis filters in terms of multipliers and adders is summarized in Table I. It can be seen that the synthesis filters with real-valued coefficients require 324 multipliers and 321 adders. On the other hand, the multiplierless realization using SOPOT coefficients requires 1058 and 1072 adders for the minimax and LS designs, respectively. After using the MB technique, the number of adders is further reduced to 508 and 529 .

Example 2: Nominal design with linear and convex quadratic constraints: In this example, we consider the constrained design with prescribed magnitude flatness and peak aliasing error. The configurations in this example are the same as those in Example 1, except that there are two constraints imposed in this example: A first-order magnitude flatness constraint is imposed at the discretized frequency points in $[0,0.05 \pi]$ to improve the reconstruction quality at low frequency, and peak-aliasing-error constraints are imposed in the frequency band $[0,0.9 \pi]$ to limit the aliasing errors to below $-100 \mathrm{~dB}$ so that the 15-bit accuracy requirement can be satisfied in this frequency band. The results so obtained are shown in Fig. 4. It can be seen from Fig. 4 that all constraints are satisfied at the expense of larger distortion ripples and aliasing errors in the frequency band $[0.9, \pi]$. This shows that there is a tradeoff between the usable bandwidth and the peak errors and, hence, the accuracy of the reconstruction systems. This example shows that the proposed algorithm offers more flexibility in error control than the traditional algorithms. This is contributed to the SOCP framework.

Example 3: Robust design with parameter uncertainties: In this example, the performances of the two robust design methods, namely, the SRD and WCRD, are evaluated. The nominal minimax design is also carried out for comparison. The filter length of digital synthesis filters are set to 81 for all the three designs. In this example, the parameters of analog analysis filters are not deterministic. In each analog analysis filter, there are four parameters that could possibly be affected with uncertainties. For simplicity, $\boldsymbol{\Phi}_{m}(j \Omega)=\left[\left(\partial H_{m}(j \Omega) / \partial l_{m, 0}\right), \ldots,\left(\partial H_{m}(j \Omega) / \partial l_{m, 4}\right)\right]^{\mathrm{T}}$, $m=1, \ldots, 4$, are set to be $[0.5,0.5,0.5,0.5]^{\mathrm{T}}$. The uncertainties are Gaussian distributed with zero mean and variance $\sigma^{2}$. In both methods, $\sigma^{2}$ is set to be 0.001 . Accordingly, approximately $68 \%, 95 \%$, and $99.7 \%$ of the parameters are within one, two, and three standard deviations of the mean. Therefore, in the WCRD method, a bound of twice the standard deviation from the mean is chosen, i.e., $\left\|\Delta \boldsymbol{l}_{m}\right\|_{2} \leq 0.002$, $m=1,2,3,4$. This contains $95 \%$ of the possible parameter
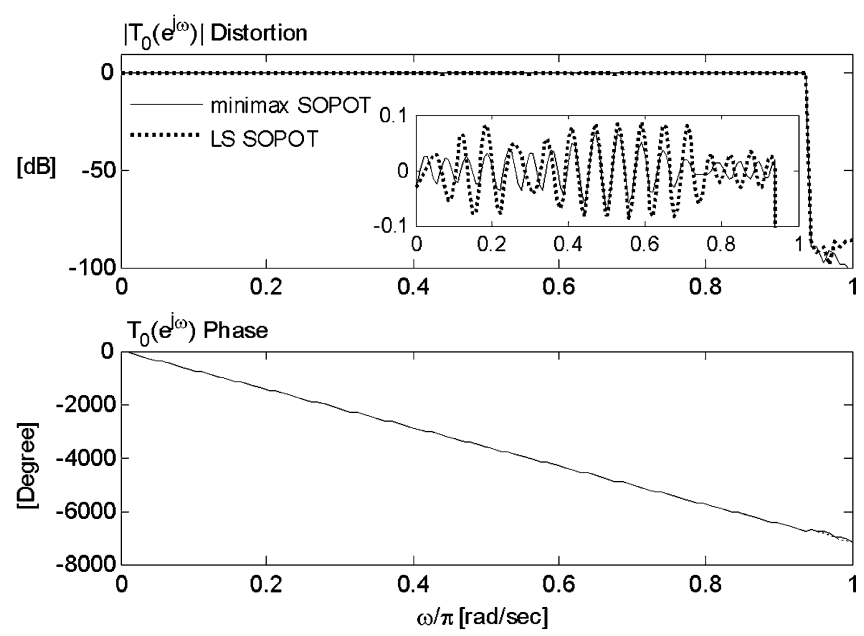

(a)
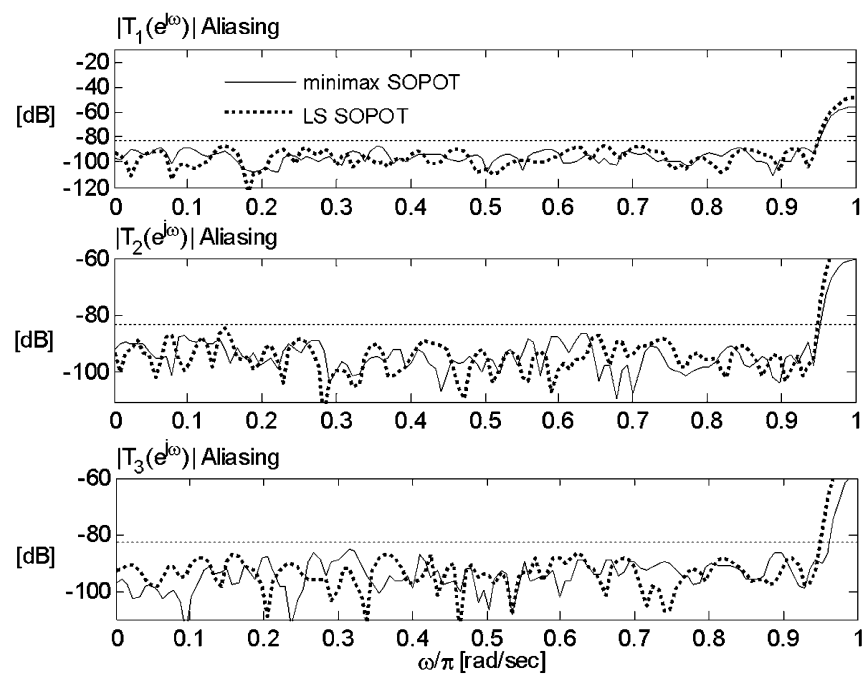

(b)

Fig. 3. LS and minimax designs using SOPOT coefficients in Example 1. (a) Distortion. (b) Aliasing errors.

TABLE I

SUMMARY OF HARDWARE COMPLEXITY IN EXAMPLE 1

\begin{tabular}{c|c|c|c|c}
\hline \multirow{2}{*}{ Criterion } & \multirow{2}{*}{ Coefficients } & \multirow{2}{*}{ Multipliers } & \multicolumn{2}{|c}{ Adders } \\
\cline { 3 - 5 } & & & Before MB & After MB \\
\hline \multirow{2}{*}{ Minimax } & Real-valued & 324 & 321 & N/A \\
\cline { 2 - 5 } & SOPOT & 0 & 990 & 462 \\
\hline \multirow{2}{*}{ LS } & Real-valued & 324 & 321 & N/A \\
\cline { 2 - 5 } & SOPOT & 0 & 1042 & 482 \\
\hline \hline
\end{tabular}

variations. The SRD and WCRD methods are then carried out, as described in Section V. Fig. 5 shows the LS approximation residue $r=\sum_{p=0}^{M-1} \int_{\Omega_{I}}\left|\bar{T}_{p}\left(e^{j \omega}\right)-D_{p}\left(e^{j \omega}\right)+\Delta T_{p}\left(e^{j \omega}\right)\right|^{2} \mathrm{~d} \omega$ of the three algorithms versus the uncertainty power $\sigma^{2} . \sigma^{2}$ is increased from 0 to 0.02 . Given $\sigma^{2}$, the parameter uncertainties are randomly generated, and the frequency responses of the three designs are then calculated according to the generated parameter uncertainties. For each given $\sigma^{2}$, this procedure is repeated 100 times to get the average performance. From 

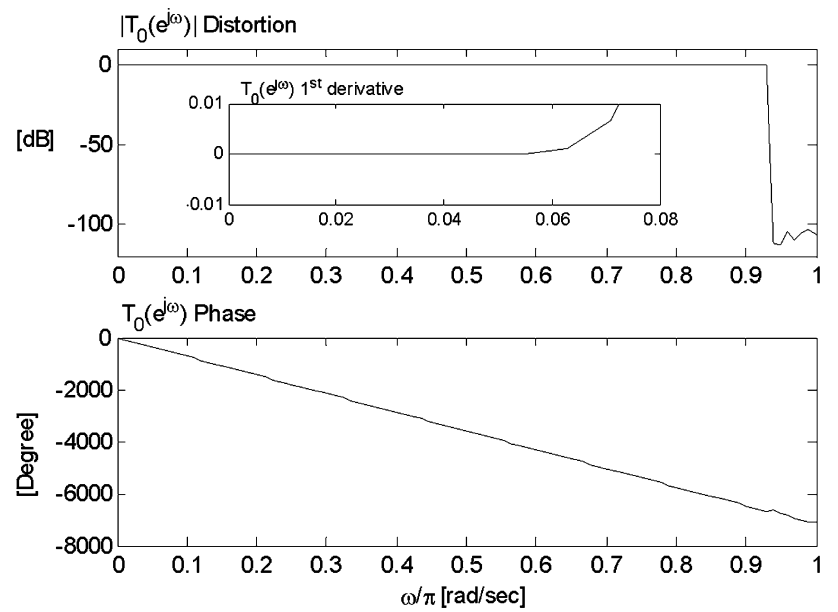

(a)
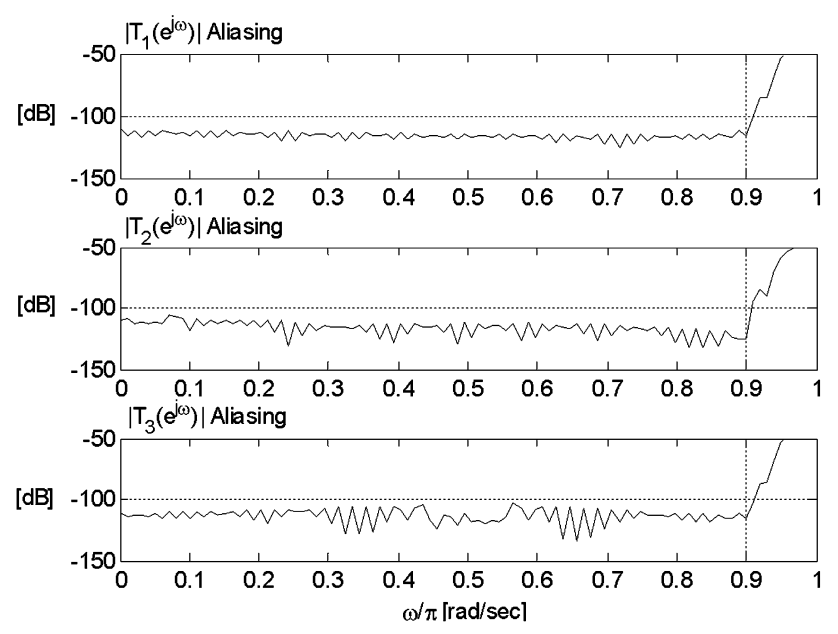

(b)

Fig. 4. Constrained design result in Example 2. (a) Distortion. (b) Aliasing errors.

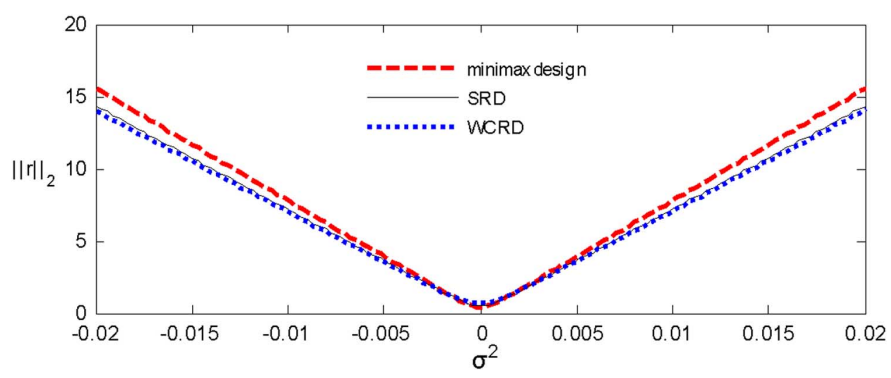

Fig. 5. LS approximation error in Example 3.

Fig. 5, we can see that when there is no parameter variation in analog analysis filters, i.e., $\sigma^{2}=0$, the minimax design gives the smallest approximation error. However, it is quite sensitive to the parameter uncertainties, and the error increases rapidly when $\sigma^{2}$ increases. The WCRD technique has a comparable but slightly inferior performance at zero noise power, while its performance in the uncertainty interval is considerably better. This is attributed to the incorporation of the parameter uncertainty model and the worst case error minimization in the algorithm.

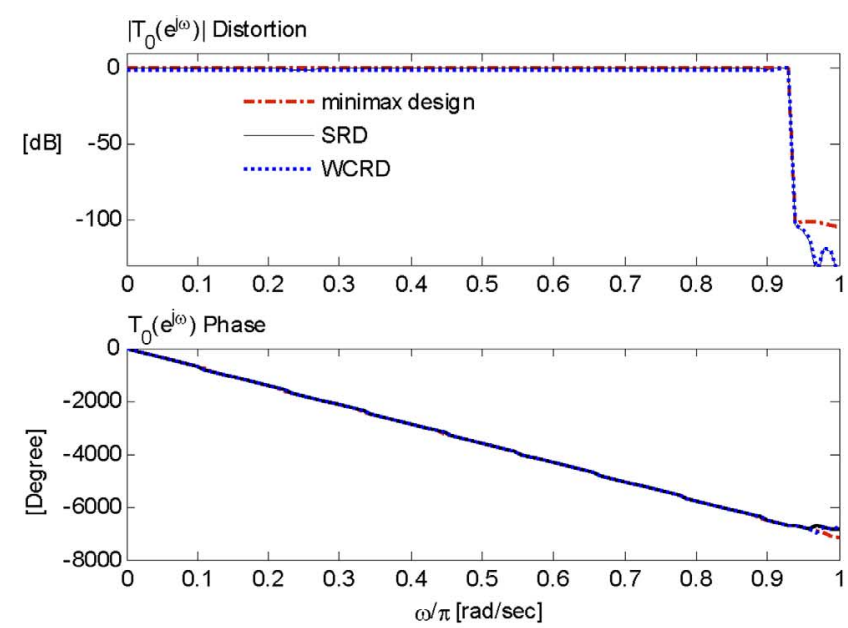

(a)
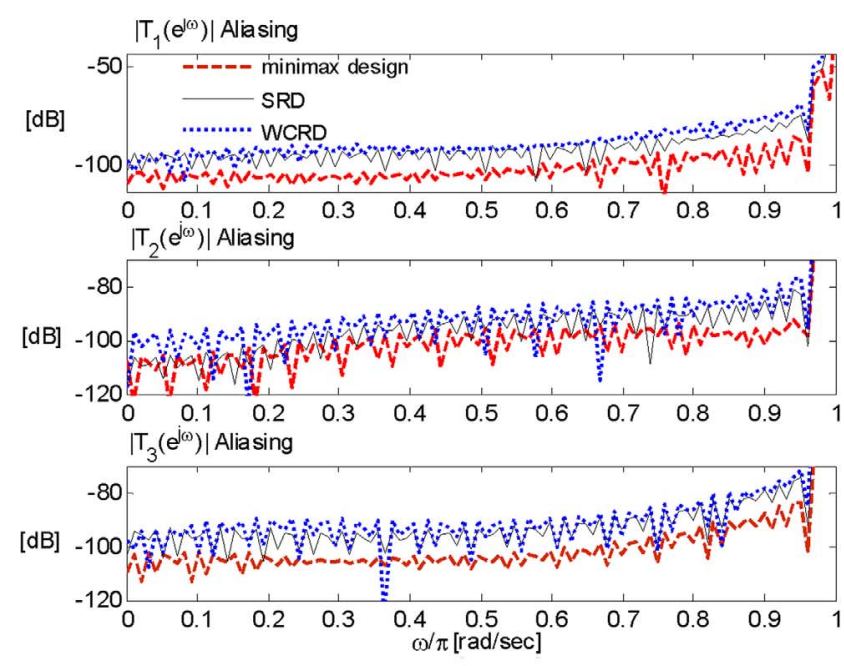

(b)

Fig. 6. Robust design with parameter uncertainties at $\sigma^{2}=0$ in Example 3 . (a) Distortion. (b) Aliasing errors.

The SRD method has a performance that is somewhat between the two. To evaluate the performance more clearly, we set $\sigma^{2}$ to 0 and 0.001 , respectively, to compare the frequency responses of the three designs. Fig. 6 shows the frequency response of the three designs when $\sigma^{2}=0$. As expected, the minimax design gives a slightly better performance than the two robust designs. Fig. 7 shows the design results when $\sigma^{2}=0.001$. Both robust design algorithms offer improved robustness to the parameter variations over the minimax design. The aliasing errors of the minimax design are suppressed to $-62 \mathrm{~dB}$. The aliasing errors of the two robust design methods are suppressed to $-66 \mathrm{~dB}$ at the desired frequency band $[0,0.95 \pi]$. The WCRD method has a slight better performance than the SRD method in this scenario.

\section{CONCLUSION}

The optimal LS and minimax designs of digital synthesis filters for HFB ADCs using SOCP have been presented. The SOCP formulation is convex, and it allows linear and quadratic constraints such as prescribed signal reconstruction magnitude 

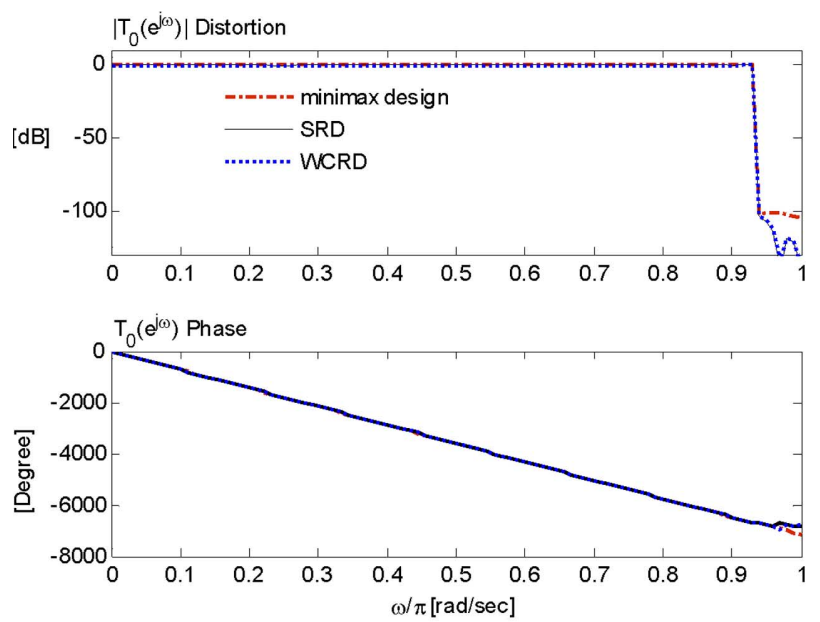

(a)
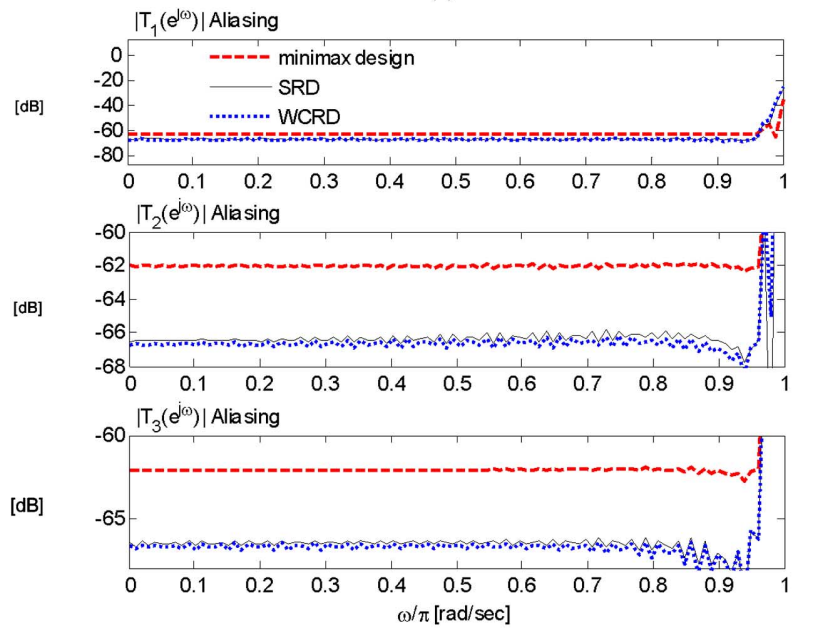

(b)

Fig. 7. Robust design with parameter uncertainties at $\sigma^{2}=0.001$ in Example 3. (a) Distortion. (b) Aliasing errors.

flatness and aliasing canceling error to be incorporated. A multiplierless hardware implementation of the proposed digital synthesis filters using SOPOT coefficients and the MB technique has been presented to minimize the hardware complexity of realization. The hardware-resource requirement was further reduced by minimizing the total internal word lengths. Two new robust design algorithms, namely, the SRD and WCRD, have been presented to reduce the sensitivity of the HFB ADC to parameter uncertainties. The designs of several reconstruction systems have also presented to illustrate the effectiveness of the proposed approaches. Design results have shown that the SOCP approach offers more flexibility than conventional methods and that the robust design algorithms are more robust to parameter uncertainties than the nominal design when the uncertainties are taken into account.

\section{REFERENCES}

[1] P. P. Vaidyanathan, Multirate Systems and Filter Banks. Englewood Cliffs, NJ: Prentice-Hall, 1993.

[2] S. R. Velazquez, T. Q. Nguyen, and S. R. Broadstone, "Design of hybrid filter bank for analog/digital conversion," IEEE Trans. Signal Process., vol. 46, no. 4, pp. 956-967, Apr. 1998.
[3] W. C. Black and D. A. Hodges, "Time interleaved converter arrays," IEEE J. Solid-State Circuits, vol. SSC-15, no. 6, pp. 1022-1029, Dec. 1980.

[4] P. Lowenborg, H. Johansson, and L. Wanhammar, "Analysis of gain and timeskew errors in filter bank based A/D converters," in Proc. IEEE Midwest Symp. Circuits Syst., Aug. 2001, vol. 1, pp. 263-266.

[5] T. Petrescu, C. Lelandais-Perrault, and J. Oksman, "Synthesis of hybrid filter banks for A/D conversion with implementation constraints-Mixed distortion/aliasing optimization," in Proc. IEEE Int. Conf. Acoust., Speech, Signal Process., May 2004, vol. 2, pp. ii-997-ii-1000.

[6] P. Lowenborg, H. Johansson, and L. Wanhammar, "A design procedure for 2-channel mixed analog and digital filter banks for A/D conversion using minimax optimization," in Proc. IEEE Int. Conf. Electron. Circuits Syst., Sep. 1999, vol. 3, pp. 1189-1192.

[7] O. Omid, "High-speed A/D and D/A converters using hybrid filter banks," in Proc. IEEE Int. Conf. Electron. Circuits Syst., Sep. 1998, vol. 1, pp. 143-146.

[8] S. Boyd and L. Vandenberghe, Convex Optimization. Cambridge, U.K.: Cambridge Univ. Press, 2004.

[9] H. Brandenstein and R. Unbehauen, "Least-squares approximation of FIR by IIR filters," IEEE Trans. Signal Process., vol. 46, no. 1, pp. 21-30, Jan. 1998.

[10] S. C. Chan, K. M. Tsui, and K. W. Tse, "Design of constrained causal stable IIR filters using a new second order cone programming based model reduction technique," IEEE Trans. Circuits Syst. II, Exp. Briefs, vol. 54, no. 2, pp. 107-111, Feb. 2007.

[11] S. C. Chan, K. M. Tsui, K. S. Yeung, and T. I. Yuk, "Design and complexity optimization of a new digital IF for software radio receivers with prescribed output accuracy," IEEE Trans. Circuits Syst. I, Reg. Papers, vol. 54, no. 2, pp. 351-366, Feb. 2007.

[12] Y. C. Lim and S. R. Parker, "FIR filter design over a discrete power-of-two coefficient space," IEEE Trans. Acoust., Speech, Signal Process., vol. ASSP-31, no. 3, pp. 583-591, Jun. 1983.

[13] C. K. S. Pun, S. C. Chan, K. S. Yeung, and K. L. Ho, "On the design and implementation of FIR and IIR digital filters with variable frequency characteristics," IEEE Trans. Circuits Syst. II, Analog Digit. Signal Process., vol. 49, no. 11, pp. 689-703, Nov. 2002.

[14] Y. C. Lim, R. Yang, D. Li, and J. Song, "Signed power-of-two term allocation scheme for the design of digital filters," IEEE Trans. Circuits Syst. II, Analog Digit. Signal Process., vol. 46, no. 5, pp. 577-584, May 1999.

[15] A. G. Dempster and M. D. MacLeod, "Use of minimum-adder multiplier blocks in FIR digital filters," IEEE Trans. Circuits Syst. II, Analog Digit. Signal Process., vol. 42, no. 9, pp. 569-577, Sep. 1995.

[16] K. S. Yeung and S. C. Chan, "The design and multiplier-less realization of software radio receivers with reduced system delay," IEEE Trans. Circuits Syst. I, Reg. Papers, vol. 51, no. 12, pp. 2444-2459, Dec. 2004.

[17] A. V. Oppenheim and R. W. Schafer, Discrete-Time Signal Processing. Englewood Cliffs, NJ: Prentice-Hall, 1989.

[18] S. C. Chan and K. M. Tsui, "Wordlength optimization of linear time-invariant systems with multiple outputs using geometric programming," IEEE Trans. Circuits Syst. I, Reg. Papers, vol. 54, no. 4, pp. 845-854, Apr. 2007.

[19] R. H. Walden, "Analog-to-digital converter survey and analysis," IEEE J. Sel. Areas Commun., vol. 17, no. 4, pp. 539-550, Apr. 1999.

[20] J. F. Sturm, "Using SeDuMi 1.02, a MATLAB toolbox for optimization over symmetric cones," Optim. Methods Softw, vol. 11/12, pp. 625-653, 1999.

[21] "The MOSEK Optimization Tools Version 2.5. User's Manual and Reference," MOSEK ApS, Copenhagen, Denmark, 2002 [Online]. Available: www.mosek.com

[22] L. B. Jackson, "Roundoff-noise analysis for fixed-point digital filter realized in cascade or parallel form," IEEE Trans. Audio Electroacoust., vol. AU-18, no. 2, pp. 107-122, Jun. 1970.

[23] L. B. Jackson, Digital Filters and Signal Processing, 3rd ed. Boston, MA: Kluwer, 1996.

[24] S. H. Zhao and S. C. Chan, "Robust design of hybrid filter bank A/D converters using second order cone programming," in Proc. IEEE Int. Symp. Circuits Syst., May 2006, pp. 891-894.

[25] S. H. Zhao, "The design of transmitter/receiver and high speed analog to digital converters in wireless communication systems: A convex programming approach," Ph.D. dissertation, Univ. Hong Kong, Hong Kong, 2009. 


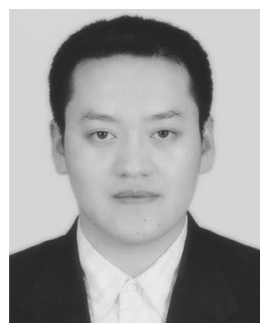

S. H. Zhao received the Ph.D. degree in electrical and electronic engineering from The University of Hong Kong, Kowloon, Hong Kong, in 2008.

He is currently a Research Associate with the Department of Electrical and Electronic Engineering, The University of Hong Kong. His main research interests include digital signal processing, convex optimization and applications, and communication signal processing.

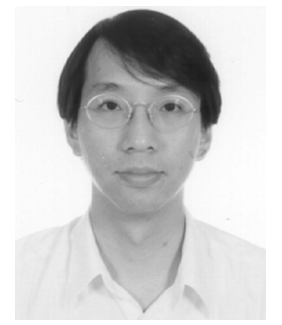

S. C. Chan (S'87-M'92) received the B.Sc.Eng. and $\mathrm{Ph} . \mathrm{D}$. degrees from The University of Hong Kong, Kowloon, Hong Kong, in 1986 and 1992, respectively.

He joined the City Polytechnic of Hong Kong in 1990 as an Assistant Lecturer and later as a University Lecturer. Since 1994, he has been with the Department of Electrical and Electronic Engineering, The University of Hong Kong, where he is currently an Associate Professor. He was a Visiting Researcher with Microsoft Corporation, Redmond, WA, and Microsoft, Beijing, China in 1998 and 1999, respectively. His research interests include fast-transform algorithms, filter design and realization, multirate signal processing, communication signal processing, and image-based rendering.

Dr. Chan is currently a member of the Digital Signal Processing Technical Committee of the IEEE Circuits and Systems Society. He was the Chairman of the IEEE Hong Kong Chapter of Signal Processing from 2000 to 2002. 